\title{
Multiphasic effect of vinyl pyrrolidone polymers on amyloidogenesis, from macromolecular crowding to inhibition
}

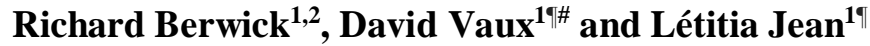 \\ ${ }^{1}$ Sir William Dunn School of Pathology, University of Oxford, Oxford OX1 3RE, UK \\ ${ }^{2}$ Current Address Royal Liverpool University Hospital, Prescot Street, Liverpool, L7 8XP
}

Running title: PVP and IAPP, from macromolecular crowding to inhibition

\begin{abstract}
II These authors are joint last authors on this work
\# To whom correspondence should be addressed: David J. Vaux, Sir William Dunn School of Pathology, University of Oxford, South Parks Road, Oxford OX1 3RE, UK.

Tel.: 0044-1865-275-544; E-mail: david.vaux@ @ath.ox.ac.uk
\end{abstract}

Key words: amyloid; type 2 diabetes; macromolecular crowding; fibril; micelle IAPP; PVP; inhibition; direct interaction, elongation

Abbreviations: AWI, air-water interface; bIAPP, biotinyl IAPP; CMC, critical micellar concentration; DMSO, dimethyl sulfoxide; HHI, hydrophobic-hydrophilic interface; IAPP, islet amyloid polypeptide; MST, microscale thermophoresis; PBS, phosphate buffer saline; PVP, polyvinyl pyrrolidone; ThT, thioflavin T; D2M, type 2 diabetes mellitus 


\begin{abstract}
Deposition of misfolded amyloid polypeptides, associated with cell death, is the hallmark of many degenerative diseases (e.g. type II diabetes mellitus and Alzheimer's disease). In vivo, cellular and extra-cellular spaces are occupied by a high volume fraction of macromolecules. The resulting macromolecular crowding energetically affects reactions. Amyloidogenesis can either be promoted by macromolecular crowding through the excluded volume effect or inhibited due to a viscosity increase reducing kinetics. Macromolecular crowding can be mimicked in vitro by addition of non-specific polymers, e.g. Ficoll, dextran and polyvinyl pyrrolidone (PVP), the latter being rarely used to study amyloid systems.

We investigated the effect of PVP on amyloidogenesis of full length human islet amyloid polypeptide (involved in type II diabetes) using fibrilisation and surface activity assays, ELISA, immuno-blot and microscale thermophoresis. We demonstrate that high molecular weight PVP360 promotes amyloidogenesis due to volume exclusion and increase in effective amyloidogenic monomer concentration, like other crowders, but without the confounding effects of viscosity and surface activity. Interestingly, we also show that low molecular weight PVP10 has unique inhibitory properties as inhibition of fibril elongation occurs mainly in the bulk solution and is due to PVP10 directly and strongly interacting with amyloid species rather than the increase in viscosity typically associated with macromolecular crowding. In vivo, amyloidogenesis might be affected by the properties and proximity of endogenous macromolecular crowders, which could contribute to changes in associated pathogenesis. More generally, the PVP10 molecular backbone could be used to design small compounds as potential inhibitors of toxic species formation.
\end{abstract}

\title{
Introduction
}

The pathology of a number of related degenerative diseases, including Alzheimer's diseases and type 2 diabetes mellitus (D2M), is strongly associated with the presence of highly ordered, fibrilar deposits of misfolded amyloid polypeptide [1]. These degenerative diseases constitute an everincreasing health concern, but presently, treatment is non-curative and focuses on patient management, not aetiology. Despite the absence of sequence homology between amyloid precursors, they exhibit similar ultrastructural and physicochemical properties with the aggregation of amyloid precursors being highly dependent upon hydrophobicity, aromatic interactions and the propensity for $\beta$-sheet formation $[2,3]$. The histo-morphological hallmark of D2M is the aggregation and deposition of a 37 amino acid peptide, human islet amyloid polypeptide (IAPP), in the pancreatic islets of Langerhans [4]. IAPP is co-expressed and secreted with insulin from the pancreatic $\beta$ cells, where it is suggested to have an endocrinological role $[5,6]$. The loss of insulin control in D2M and accumulation of IAPP aggregates are coterminous with the loss of $\beta$ cell mass $[7,8]$.

Amyloidogenesis is a polymerisation-driven process composed of three major steps: an energetically unfavourable nucleation (lag phase) leading to the formation of a minimal self-assembled nucleus (oligomer), elongation of nuclei by monomer addition until the system reaches equilibrium between fibrils and monomers (plateau)[9]. Amyloid fibrilogenesis can be catalysed by hydrophobichydrophilic interfaces (HHIs) due to the amphiphilicity and surfactant properties of amyloid polypeptides [10-17]. HHIs catalyse assembly into fibrils by promoting peptide chains alignment in which polar and non-polar side-chains segregate on opposite sides of the $\beta$-strand, spatially concentrating polypeptides and altering the thermodynamic equilibrium. Phospholipid membranes, the ultimate in-vivo HHI, are known to catalyse amyloidogenesis, which in turn may lead to membrane damage [12,18-22]. Amyloid toxicity towards membrane is suggested to be due to various mechanisms, such as pore forming oligomers, rupture of membranes through monomer insertion and subsequent elongation [20-23]. IAPP interacts with $\beta$ cell membranes, which concomitantly increases membrane permeability and can lead to calcium dysregulation [24]. The mechanisms of IAPP interaction with $\beta$ cell membrane are thought to be prototypical of many other protein misfolding diseases, such as Alzheimer's and Parkinson's diseases [25].

Despite the knowledge that amyloid species are surface active and accumulate at HHIs, most in-vitro assays are performed in the presence of an air-water interface (AWI; non-polar gas and polar aqueous solution). The critical importance of the AWI on all kinetic parameters of IAPP fibrilogenesis has previously been demonstrated [16]. The effect of AWI removal was dependent on the initial IAPP 
monomer concentration and was manifest as an absence of fibrilogenesis below a certain initial monomer concentration. Moreover, the enhancing effect of lipids on IAPP amyloidogenesis was shown to be at its greatest in a context more closely mirroring in-vivo conditions, i.e. in the absence of an AWI. The existence of two separate pathways for nucleation was proposed, one dependent on monomer adsorption to the AWI, and one dependent on micelle (or intermediate) formation in the bulk solution (Figure 1)[16, 26]. Like any other surfactant, amyloids form micelles only above their critical micellar concentration (CMC). These are the species responsible for the subsequent formation of nuclei in the bulk solution. Below the CMC, the only nuclei formed are at the AWI. Therefore, the AWI dependent pathway to fibrilar species is very rapid. Thus, in the absence of an AWI and above the CMC, nucleation would solely rely on micelle formation in the bulk solution, which is slow due to the absence of a surface for peptide orientation and alignment. In contrast, in the absence of an AWI and below the CMC, nucleation would not happen on a biologically relevant time scale. Although the AWI does not fully encompass the complexity of in-vivo HHIs, it represents a very homogeneous and reproducible in-vitro model of an HHI. Maintaining the AWI in in-vitro assays allows the study of the influence and role of a model HHI on amyloidogenesis, whereas its removal allows the study of more physiologically relevant bulk system.

Macromolecules occupy a large fraction of the intracellular and extracellular environments, resulting in a fraction of the volume being unavailable for other molecules to occupy [27, 28]. This 'excluded volume effect' is also known as macromolecular crowding, whereby the effective concentration of molecules within a defined volume is increased due to the same molar amount of the molecules being present in a much smaller volume. This crowding can have large energetic consequences on reaction rates and equilibria, such as shifting the equilibrium of species and leading to the promotion of macromolecular aggregation [28-30]. Crowded conditions found in cells can be mimicked, in-vitro, by the addition of non-specific model crowding agents (e.g. Ficoll and dextran)[28, 31]. The effect of macromolecular crowding on amyloidogenesis is complex. Firstly, the excluded volume effect should promote aggregation due to an increase in effective monomer concentration, which should increase the probability of aggregation-prone associations [32-36]. Secondly, the increase in viscosity introduced by some crowding agent should reduce the diffusion rate of monomers, initially to other monomers, and then later to oligomers and fibril. As the concentration of crowding agent increases, there would be increasingly inhibitory effects of viscosity as the reaction becomes diffusion-limited $[32,36]$. At very high polymer concentrations this would outweigh the accelerating effects of the increased effective concentration. In the case of $\mathrm{A} \beta$, a major key player in Alzheimer's disease pathogenesis, we indeed demonstrated previously that the excluded volume effect dominates over viscosity in a crowder dosedependent manner under quiescent conditions. Furthermore, we showed that the nature and properties of the crowding agent also influences the assembly process, with dextran promoting through volume exclusion until viscosity impedes it, whereas Ficoll inhibits assembly due to surface activity and increased viscosity despite volume exclusion. Under non-quiescent conditions, Ficoll and dextran were also found to inhibit IAPP amyloidogenesis [37]. Thus, it is clear that macromolecular crowding by addition of any synthetic crowder is not a straightforward process as the size, viscosity, surface activity and inertia (non-protein binding capacity) will each play significant roles. Polyvinyl pyrrolidone (PVP) is another type of macromolecular crowding agent, which has very rarely been used on amyloid systems, with only one study showing both promotion and inhibition of insulin amyloidogenesis by PVP [38]. Therefore, the effect of PVP on amyloid assembly is not a well-established concept. Nonetheless, PVP has advantageous characteristics such as exquisite wetting properties, enhanced cross-linking, stability, non-toxic, uncharged, and protein-like solution properties [39]. In contrast to other commonly used crowders (e.g. dextran and Ficoll), PVP is a non-carbohydrate polymer, which has minimum viscosity and surface activity. This is an important distinction, since crowding can be an interplay between the excluded volume effect and viscosity (e.g. dextran), and/or between the excluded volume effect, surface activity and viscosity (e.g. Ficoll). Therefore, the use of high molecular weight PVP allows the study of the effect of volume exclusion without any interference from viscosity or surfactant effects. Whereas, the use of low molecular weight PVP allows the study of the relationship between polymer size and effects on assembly reactions. In this study, we investigated the effect of macromolecular crowding by PVP on full-length human IAPP adsorption at the AWI and fibrilisation. Here, we show that the effect of macromolecular crowding by PVP is dependent on the chain length of PVP (i.e. molecular weight), with high molecular weight PVP360 promoting IAPP assembly via the excluded volume effect. These 
result are confirmatory of the effect on amyloid assembly of macromolecular crowding as far as volume exclusion is involved but differ to previous results obtained with dextran and Ficoll, as no inhibition of IAPP fibrilisation was observed with PVP360 due to the absence of viscosity increase and surface activity effect. Furthermore, we demonstrate that low molecular weight PVP10 interacts strongly with IAPP species to sequester them in an elongation incompetent form, leading to an inhibitory effect on IAPP assembly that is more prominent in the bulk solution.

\section{Experimental}

\section{Peptide and reagents}

$\mathrm{AChE}_{586-599}$ was synthesised and prepared as previously described [40]. Lyophilised synthetic human IAPP and biotinyl full-length human IAPP (Bachem, Germany) were purchased already purified by reverse-phase high performance liquid chromatography and were dissolved in dimethylsulfoxide (DMSO) at 512.4 and $128.1 \mu \mathrm{M}$ respectively. To remove pre-aggregated species, samples were sonicated and centrifuged at $15,000 \mathrm{~g}$ for 1 hour, at $4^{\circ} \mathrm{C}$ prior to use. DMSO was used to maintain the peptides in a monomeric pool lacking any $\beta$-sheet secondary structures [41]. Stock solution of 1 or 10 $\mathrm{mM}$ of $10 \mathrm{kDa}$ PVP (PVP10), $640 \mu \mathrm{M}$ of $360 \mathrm{kDa}$ PVP (PVP360), 10\% BSA (Sigma-Aldrich) were prepared in distilled water.

\section{Fibrilisation Experiments}

Several concentrations of IAPP were incubated with $32 \mu \mathrm{M}$ thioflavin T (ThT) in PBS, in presence or absence of various molecular weights of PVP. $125 \mu \mathrm{M} \mathrm{AChE}_{586-599}$ was incubated with $165 \mu \mathrm{M}$ thioflavin T (ThT) in PBS, in presence or absence of various concentrations of PVP10. Measurements of ThT fluorescence (excitation $450 \mathrm{~nm}$, emission $480 \mathrm{~nm}$ ) were taken in a 96 well plate (black wall, clear bottom; Greiner Bio-One, UK) on a Polarstar plate reader (BMG Labtech, UK), at $37^{\circ} \mathrm{C}$, using a bottom-bottom configuration (optical fiber system detecting emission signal from the bottom of the well) and under non-agitating conditions for IAPP and very mild agitating conditions ( $5 \mathrm{~s}$ every $66 \mathrm{~s}$ ) for $\mathrm{AChE}_{586-599}$. To remove the AWI, Perspex cylinders were introduced at the start of the reaction, as previously described [17]. The control wells (buffer with or without cylinder/PVP) were subtracted from the test values (peptide with or without PVP). The lag phase was obtained from the intercept on the time axis of the line formed tangent to the inflection point [42]. The elongation rate was obtained from the slope at the inflection point of the sigmoidal curve and the plateau height from an average of the highest curve values attained at the end of the experiment $[42,43]$. To facilitate direct comparison between ThT fibrilisation assays, the plateau height was then normalised to the highest fluorescence value reached by $1.82 \mu \mathrm{M}$ IAPP alone in presence of an AWI (as the only condition common to all fibrilisation experiments). In the case of a biphasic behaviour for the elongation, the rate was calculated from the slope at the second inflection point (i.e. not for the first but for the second increase in elongation). At least three independent assays were performed. Results were analysed with the twosample t-test.

\section{Surface activity measurement}

The DMSO stock solution of IAPP, in absence or presence of $5 \mu \mathrm{M}$ PVP10, was diluted to $1.2 \mu \mathrm{M}$ in $80 \mu \mathrm{l} 200 \mathrm{mM}$ sodium acetate $\mathrm{pH} 3$ and $20 \mu \mathrm{l} 1 \mathrm{M} \mathrm{NaH}_{2} \mathrm{PO}_{4} \mathrm{pH} 7$ in wells of a 96-well plate (black wall, clear bottom; Greiner Bio-One). Surface activity was measured, repeatedly at one minute intervals, at $450 \mathrm{~nm}$ on a BMG Polarstar plate reader at the central and offset positions, as previously described [11]. Control values (DMSO with or without PVP10) were subtracted to all test values (IAPP with or without PVP10). $\triangle \mathrm{OD}=\left(\mathrm{OD}_{\text {offset position }}-\mathrm{OD}_{\text {central position }}\right)$. At least three independent assays were performed and analysed with the two-sample t-test.

\section{ELISA}

Wells of a 96-well plate (black wall, clear bottom; Greiner Bio-One, UK) were incubated overnight in PBS with $100 \mu \mathrm{l}$ of $428 \mu \mathrm{M}$ or $5 \mu \mathrm{M}$ PVP10, or $10 \mu \mathrm{M}$ PVP360, or 5\% BSA, or no polymer. The wells were then incubated with $70 \mu \mathrm{l}$ of $3.6 \mu \mathrm{M}$ IAPP and $0.4 \mu \mathrm{M}$ bIAPP, or PBS (no IAPP control), for $5 \mathrm{~h}$ at room temperature, before incubation with $70 \mu \mathrm{l}$ of avidin Texas red, or PBS (no avidin control) for 30 min. Texas red fluorescence was measured on a BMG Fluostar Optima plate reader (excitation 550 
$\mathrm{nm}$, emission $600 \mathrm{~nm}$ ). Three independent assays were performed and analysed with the two-sample ttest.

\section{Immuno-blot}

Strips of nitrocellulose were incubated overnight in PBS with $428 \mu \mathrm{M}$ or $5 \mu \mathrm{M}$ PVP10, or $10 \mu \mathrm{M}$ PVP360, or 5\% BSA, or no polymer. The strips were then incubated with $3.6 \mu \mathrm{M}$ IAPP and $0.4 \mu \mathrm{M}$ bIAPP, or PBS (no IAPP control), for $5 \mathrm{~h}$ at room temperature, before incubation with avidin HRP (Vector Elite ABC, Vector Laboratories), or PBS (no avidin control) for $30 \mathrm{~min}$. HRP activity was detected by using DAB according to manufacturer instructions (Vector Laboratories). The intensity of the DAB coloration was quantified by image analysis in FIJI (ImageJ 1.51n).

\section{Microscale thermophoresis (MST)}

We used bIAPP and avidin fluorescein as a probe for monitoring thermophoretic movement. $3.6 \mu \mathrm{M}$ IAPP, $0.4 \mu \mathrm{M}$ bIAPP, $0.08 \mu \mathrm{M}$ avidin fluorescein were mixed in PBS and $0.05 \%$ Tween 20 , in presence of increasing concentrations of PVP10. Each solution was immediately transferred to a glass capillary (standard treatment, NanoTemper Technologies MO-K002) and immediately transferred to the instrument. The measurements were performed at $25^{\circ} \mathrm{C}$ on a Monolith NT.115 (NanoTemper Technologies), with an infrared laser power of $40 \%$ (to create the temperature gradient by focal heating of the solution) and the blue LED channel with $3 \%$ power (for fluorescein excitation). Three capillaries were used per conditions, and at least three independent assays were performed per conditions. Normalised fluorescence (Fnorm) was obtained from raw fluorescence using the Nanotemper Analysis software 1.5.41. For Kd determination, all curves of Fnorm values from analysis of the combined thermophoresis $+\mathrm{T}$ jump, were plotted using GraphPad Prism v7.02. Fitting and analysis were carried out in GraphPad Prism using non-linear regression and [inhibitor] vs response-3 parameters.

\section{Results \\ PVP360 promotes IAPP fibrilisation}

Non-carbohydrate polymers, such as polyethylene glycol and PVP are commonly used as macromolecular crowders. PVP is a random coil $\gamma$ lactam polymer, which is an important blood plasma substitute in trauma medicine $[44,45]$. However, PVP has very rarely been used as a macromolecular crowder of amyloid systems [38]. Shaking is often used to accelerate amyloidogenesis but may not represent a physiologically relevant condition. Therefore, we performed our experiments in quiescent conditions. The concentration of IAPP used is $1.82 \mu \mathrm{M}$, which is just above IAPP CMC. IAPP CMC was proposed to lie between 1.3 and $1.8 \mu \mathrm{M}$ [16]. Below the CMC, nuclei are only formed at the AWI (Figure 1). In contrast, above the CMC nuclei are formed both at the AWI and from micelles in the bulk solution. $1.82 \mu \mathrm{M}$ was chosen as it is the lowest IAPP concentration allowing rapid fibrilisation assays as nucleation can take place both at the AWI and from micelles in the bulk solution.

We first tested the effect of a high molecular weight PVP, PVP360, which should promote macromolecular crowding through the excluded volume effect. In order to follow the formation of IAPP fibrils, we used the typical amyloid dye, thioflavin T (ThT) which changes its fluorescence emission from 450 to $480 \mathrm{~nm}$ when intercalated into $\beta$ sheet [46]. Nucleation (lag phase) of $1.82 \mu \mathrm{M}$ IAPP was only significantly accelerated by $160 \mu \mathrm{M}$ PVP360 (Figure 2 and Supplementary Figure S1). In contrast, all concentrations of PVP360 significantly increased the plateau height (by 1.7 to 3.9 fold), whereas only 5 to $160 \mu \mathrm{M}$ significantly increased the elongation rate (by 1.1 to 1.9 fold). Thus, PVP360 acted as a macromolecular crowder in promoting IAPP fibrilisation.

\section{PVP10 inhibits IAPP fibrilisation}

Having demonstrated that PVP360 can act as a macromolecular crowder, we then investigated whether the promoting effect on IAPP fibrilisation was dependent only on PVP 'bulkiness' (i.e. molecular weight), i.e. by the excluded volume effect. To assess this, we repeated fibrilisation experiments with PVP10, a PVP species that should not promote the excluded volume effect due to its lower molecular weight and smaller Stokes radius.

Nucleation of $1.82 \mu \mathrm{M}$ IAPP was not affected by any of the PVP10 concentrations (Figure 3A and B). In contrast to PVP360, all concentrations of PVP10 significantly reduced IAPP elongation rate 
(by 54 to $85 \%$ ) and plateau height (by 18 to $49 \%$, except 0.04 and $0.16 \mu \mathrm{M}$ ) (Figure 3). Pairwise comparison between the inhibitory effect of $5 \mu \mathrm{M}$ PVP10 and that of the other PVP10 concentrations reveals that only the lowest two PVP10 concentrations $(0.08$ and $0.04 \mu \mathrm{M}, P<0.045$ and $P<0.03$ respectively) have significantly less inhibitory effect. This suggests that for the inhibition of elongation, the maximum effect size has already been reached by $0.16 \mu \mathrm{M}$, with only PVP10 concentrations lower than this having significantly smaller effects.

Thus, it is clear that the effect of PVP on IAPP assembly is molecular weight dependent, with PVP10 inhibiting and PVP360 promoting.

\section{PVP10 also inhibits another amyloidogenic peptide, AChE $_{586-599}$}

We then assessed whether PVP10 inhibition was specific or not to IAPP by using another amyloid system. We used a peptide from the C-terminal oligomerisation domain of the synaptic form of human acetylcholinesterase, $\mathrm{AChE}_{586-599}$. This peptide has previously been shown to undergo a conformational switch from random coil to $\beta$-sheet, to be surface active and to form typical amyloid fibrils [47].

As for IAPP, nucleation of $\mathrm{AChE}_{586-599}$ was not affected by PVP10 but the elongation rate and plateau height were (Figure 4). Indeed, the elongation rate was significantly inhibited by $0.8,420$ and $100 \mu \mathrm{M}$ PVP10 (from 1.7 to 4.7 fold inhibition), and the plateau height was significantly inhibited by 4, 20 and $100 \mu \mathrm{M}$ PVP10 (from 1.2 to 2.2 fold inhibition).

Therefore, PVP10 inhibitory effect is not specific to IAPP and might be generic.

\section{PVP10 reduces IAPP adsorption to the AWI}

To dissect the PVP10 mode of inhibition, we next assessed whether it was influencing the recruitment of IAPP monomers at the AWI. For this, we qualitatively measured the dynamics of surface activity of IAPP precursor species in absence or presence of PVP10 (Figure 5). We used a previously described technique based on an off-axial light beam to measure the lensing effect of a meniscus [11]. The principal behind this technique is that a surfactant induces a meniscus curvature, which is proportional to the surfactant activity. Normalising the apparent optical density measured at the offset using the optical density measured on the central axis $(\Delta \mathrm{OD})$ gives a value strongly inversely correlated with surface tension $(\mathrm{R}>0.97)$. This technique allows qualitative surface activity measurements over longer time course analysis than traditional quantitative surface tension measurements (e.g. drop shape analysis, during which the drop detached itself from the tip of the needle due to the high surface activity of amyloid and liquid evaporation from the drop becomes too important over time to exclude any peptide concentration effect on surface tension).

We used $1.2 \mu \mathrm{M}$ IAPP, which is believed to be below IAPP CMC, in order to avoid any interference from bulk micelles, and to maximise IAPP recruitment at the AWI. The measurements were performed at the beginning of a fibrilisation reaction in order to assess the surface activity of amyloid precursor species. IAPP adsorption at the AWI was constant with a high $\triangle$ OD being observed, indicative of a high surface activity, as previously observed [17]. By itself, PVP10 initially showed a $\triangle \mathrm{OD}$ half of that of IAPP, which then very rapidly declines (within the first $10 \mathrm{~min}$ ) and levels off at the baseline level for water alone. Therefore, PVP10 displays a very rapid and modest surfactant activity when first introduced to the well. In the presence of PVP10, IAPP surface activity was significantly reduced by $7.5 \%(P<0.032)$, suggesting that PVP10 slightly reduces IAPP adsorption to the AWI.

\section{PVP10 also inhibits IAPP assembly in the bulk solution}

We next wanted to probe PVP10 mode of inhibition further by assessing whether it is affecting IAPP assembly only at the AWI and/or in the bulk solution. For this, we performed fibrilisation assays across a series of IAPP concentrations using a single concentration of PVP10 both in the presence and absence of an AWI. The AWI was removed by introducing hydrophilic Perspex cylinders, as previously described [16, 17]. Perspex is very hydrophilic and the new Perspex-solution interface created replaced the AWI HHI by a hydrophilic-hydrophilic interface. In absence of an AWI, fibrilisation relies solely on micelle formation in the bulk solution, which would not happen at IAPP concentration below the CMC. $5 \mu \mathrm{M}$ PVP10 was chosen as it was the concentration that showed the highest percentage of inhibition for both elongation rate and plateau height (Figure 3). We used IAPP concentrations spanning 
below $(1,1.2 \mu \mathrm{M}$ and $1.5 \mu \mathrm{M})$, around $(1.82 \mu \mathrm{M})$ and above CMC (4 and $12 \mu \mathrm{M})$, which should provide further information on PVP10 action on micelle formation. So, by altering the IAPP concentration, we are directly influencing the micelle concentration in a dose-dependent manner.

In the presence of an AWI, as seen in Figure 3 for $1.82 \mu \mathrm{M}$ IAPP, PVP10 did not affect the lag phase of any IAPP concentration tested (Figure 6A and Supplementary Figure S2). The plateau height was not significantly affected by PVP10 for all IAPP concentrations tested, except for $1.82 \mu \mathrm{M}$ IAPP for which a significant inhibition was seen $(P<0.0019)$, as previously observed in Figure 3 (Figure $6 D$ ). In contrast, PVP10 significantly reduced the rate of elongation across all IAPP concentrations tested (except for $1 \mu \mathrm{M}$ IAPP)(Figure 6A and $\mathrm{C}$, grey versus black non-filled bars). However, for IAPP concentrations below and above CMC, the inhibitory effect decreased as the IAPP concentration increased, with 1.63 fold inhibition for 1 and $1.2 \mu \mathrm{M}$ IAPP, 1.43 fold for $1.5 \mu \mathrm{M}, 1.34$ fold for $4 \mu \mathrm{M}$, 1.21 fold for $12 \mu \mathrm{M}$. Thus, flooding the system with increasing amount of IAPP monomers was 'diluting' the inhibition.

In contrast, and as previously observed, the removal of the AWI at IAPP concentrations below $\mathrm{CMC}$ resulted in the absence of fibrilogenesis (1 and $1.2 \mu \mathrm{M}$,)(Figure 6B)[16, 17]. At 1.5 $\mu \mathrm{M}$ IAPP, approaching the CMC, very minimal assembly was detected (Supplementary Figure S2). Also, as previously observed in the absence of an AWI, a significant reduction in plateau height was found for all IAPP concentrations tested, when compared to assays in presence of an AWI, showing that the final amount of amyloid fibril at equilibrium critically depends on whether the AWI is present or not (Figure $6 \mathrm{~B}$ and D, grey versus purple bars)[16, 17]. For $1.82 \mu \mathrm{M}$ IAPP, around the CMC, PVP10 inhibited both the elongation rate and plateau height, as observed in presence of an AWI $(P<0.012$ and $P<0.05$, respectively). Moreover, irrespective of the presence or absence of an AWI, PVP10 also significantly inhibited the elongation rate for IAPP concentrations above the CMC. In the absence of an AWI the inhibitory effect on elongation decreased, with the effect decreasing as the IAPP concentration increased above the CMC, with 2.37 fold inhibition for $4 \mu \mathrm{M}$ and 1.36 fold for $12 \mu \mathrm{M}$ (Figure $6 \mathrm{~B}$ and $\mathrm{C}$, purple versus purple non-filled bars). In contrast to in presence of an AWI, the plateau height was also significantly decreased by PVP10 at IAPP concentration above the CMC, with again a reduction of the inhibition as the IAPP concentration increased (1.71 fold for $4 \mu \mathrm{M}$ and 0.94 fold for $12 \mu \mathrm{M}$ ).

Altogether these results strongly suggest that PVP10 preferentially inhibits IAPP elongation, and that it is more active in the bulk solution than at the AWI.

\section{PVP10 interacts with IAPP}

To assess whether PVP10 was inhibiting IAPP fibrilisation through direct interaction or not, we performed three different tests, ELISA, immuno-blot and microscale thermophoresis.

For the ELISA, we assessed $5 \mu \mathrm{M}$ PVP10 (which is the most inhibitory concentration), $10 \mu \mathrm{M}$ PVP360 (which is one of the lowest promoting concentration), alongside 5\% BSA as a control. All of these were coated onto the wells overnight. To report IAPP binding, we used biotinyl IAPP (bIAPP) as a spike, 1:9 ratio with IAPP, and avidin Texas red. $4 \mu \mathrm{M}$ IAPP overall $(3.6 \mu \mathrm{M}$ IAPP with $0.4 \mu \mathrm{M}$ bIAPP) was chosen as it is one of the highest IAPP concentration, above CMC, for which elongation was inhibited by PVP10 (see Figure 6). The polymer coated wells, or control wells without polymer, were incubated with bIAPP:IAPP for $5 \mathrm{~h}$, to allow the reaction to be in the elongation phase. Therefore, it allowed us to assess a range of IAPP species involved in binding to PVP10, including monomers, micelles, nuclei and protofibrils. bIAPP:IAPP by themselves showed non-specific binding to the plastic of the well (Figure 7A, last two columns). However, this should have been minimised in the test wells as $100 \mu \mathrm{l}$ polymer solution was applied, which should have coated not only the bottom of the well but also the walls, and then only $70 \mu \mathrm{I}$ IAPP and avidin solutions were applied. Avidin Texas red also nonspecifically bound to the polymer-coated well (third, fifth and seventh columns). Nonetheless, the signal for IAPP binding to PVP10 (first column) was clearly specific as significantly higher than that of avidin Texas red non-specifically binding to PVP10 (third column, $\sim 1.2$ fold, $P<0.008$ ), and than that of bIAPP:IAPP non-specific binding to the plastic (penultimate column, 2.2 fold higher, $P<4 \times 10^{-7}$ ). In contrast, despite being higher than that of bIAPP:IAPP non-specific binding to the plastic (penultimate column), the signal found for PVP360 in presence of bIAPP:IAPP and avidin Texas red (fourth column) was no different to that of avidin Texas red non-specifically binding to PVP360 (sixth column). bIAPP:IAPP did not interact with our control coated well, 5\% BSA (seventh to ninth column), despite 
BSA being known to inhibit fibril formation of some amyloids by direct binding, either tightly or weakly depending on the amyloid (e.g. A $\beta$, insulin, and transthyretin) [48-51]. Our ELISA clearly shows that IAPP binds to PVP10 coated wells but not to PVP360 or 5\% BSA coated wells.

We confirmed a specific interaction of PVP10 with IAPP by immuno-blot. Similarly to the ELISA, both bIAPP:IAPP and avidin HRP by themselves bound non-specifically to the nitrocellulose or polymers respectively (Figure 7B and C, last 2 columns). However, the blots clearly show that IAPP bound specifically to PVP10 as the intensity of DAB reaction was $\sim 1.4$ fold stronger than that of IAPPavidin non-specifically binding to the nitrocellulose (first column versus penultimate column in C). The intensity of DAB reaction for IAPP binding to PVP360 or 5\% BSA was no different than that of avidin non-specifically binding to the polymer (third versus fifth, and sixth versus seven column).

Thus, these two different types of experiment independently show that only PVP10 is able to specifically interact with IAPP.

\section{PVP10-IAPP interaction, Kd determination}

To determine the strength of PVP10-IAPP interaction, we used microscale thermophoresis (MST). This recent biophysical method monitors the direct movement of molecules along a temperature gradient according to their size, charge, and hydration shell, with molecular interactions affecting at least one of these parameters [52-54]. Indeed, depending on conformational changes induced by the formation of a complex between a molecule and its partner, one of which being fluorescently labelled or intrinsically fluorescent, the thermophoresis of the molecule alone and that of the complex can differ significantly. MST is a very sensitive technique allowing the study of molecular interactions under steady-state conditions. Any changes in thermophoresis of the labelled molecule reflect changes that affected the thermophoretic mobility and diffusion coefficient, and are indicative of a global change of this molecule. Binding is detected by a quantification of the change in the normalised fluorescence. MST has been previously successfully used to detect binding of inhibitors to IAPP ${ }_{11-20}$ and other amyloids [55-57]. To monitor IAPP, we used bIAPP at a 1:9 ratio with IAPP ( $4 \mu \mathrm{M}$ IAPP overall), and avidin fluorescein. The measurements were carried out immediately after mixing, i.e. $t=0$, to investigate only IAPP-PVP10 induced changes rather than IAPP aggregation induced changes, and therefore the binding is most likely to occur to IAPP monomers or small oligomers. Fig. 8A shows normalised fluorescence time traces of IAPP alone and IAPP with $0.08 \mu \mathrm{M}$ PVP10. As shown on this figure panel, a typical MST trace comprises three time intervals; before, during and after induction of the temperature gradient. Before induction represents the fluorophore initial state. During induction corresponds to the temperature jump during which fluorescence changes due to sample heating, and thermophoresis during which fluorescence changes due to thermophoretic motion. After induction represents the inverse temperature jump induced by sample cooling, and back diffusion during which the fluorescence recovers due to molecule mass diffusion. Each of these phases contain information about binding affinity and mechanism. It is evident that the time trace for IAPP alone was different to that in presence of $0.08 \mu \mathrm{M}$ PVP10, indicating a PVP10-induced change in the thermophoretic movement of fluorescein, which is statistically significant from $\sim 5.7 \mathrm{sec}$ until $36 \mathrm{sec}$, i.e. the whole of the thermophoretic movement. This is clear evidence that PVP10 binds to IAPP as the thermophoresis of the complex differed from that of IAPP alone. It was previously proposed that if the temperature jump signal is unaffected or minimally affected, but the thermophoresis signal is significantly affected, this is indicative of the properties of the entire molecule-complex being affected, rather than just the local surrounding of the fluorophore [54]. Therefore, binding of PVP10 is more than likely to affect IAPP rather than just fluorescein.

Figure $8 \mathrm{~B}$ shows normalised fluorescence traces of IAPP in presence of increasing concentrations of PVP10 in order to determine the Kd. We observed a decreasing sigmoidal MST signal with increasing PVP10 concentration, with a good thermophoretic amplitude of $\sim 57$ units (Fnorm starting at $\sim 900$ and decreasing to $\sim 843$ units). The Kd for PVP10 binding to IAPP was found to be $1.14 \pm 0.54 \mu \mathrm{M}$. Therefore, PVP10 interacts strongly with IAPP monomers, with a Kd smaller than that of self-aggregating IAPP ${ }_{11-20}$ in PBS $(6.56 \mu \mathrm{M})$ [58]. This suggests that PVP10 may inhibit IAPP assembly by weakening the interaction of IAPP with itself, resulting in reduced self-aggregation. 
PVP10 might compete with IAPP monomers during the aggregation process and therefore inhibit fibril formation. Our binding curve showed a negative slope, i.e. a decrease in Fnorm with increasing PVP10 concentrations. MST binding responses can typically be positive or negative, as in our case [59]. Indeed, similar decrease in Fnorm was also observed for other amyloid systems upon binding of small peptides/molecules (e.g. IAPP ${ }_{11-20}$ or tau fibrils,) but also for non-amyloid systems (e.g. the Ser/Thr kinase p38 and its inhibitor BIRB-796, SNARE-liposome complexes, and the $\beta$-lactamase TEM-1 with its inhibitor BLIP) $[55,56,59,60]$. In the case of TEM-1, it was suggested to be due to a conformational change of TEM-1 when bound by BLIP.

To assess whether IAPP contained binding sites for PVP10 with different cooperativity, we analysed further our data in GraphPad Prism using a non-linear regression and [inhibitor] vs response under two fitting conditions, i.e. using 3 parameters with a fixed Hill slope of 1 (one type of binding site), or 4 parameters with a non-fixed Hill slope (cooperativity, different types of binding sites). For each data set, we selected the Hill slope, set it to the hypothetical value of 1 , and assessed whether the best fit value differed from the hypothetical value. We found a p-value of 0.8412 for the null hypothesis that the Hill number is different from 1 using an F-test.

Thus, together these results demonstrate that PVP10 binds strongly to IAPP using only one type of binding site(s).

\section{Discussion}

Amyloidogenesis is usually studied under dilute conditions not reflecting the in vivo crowded conditions of the cell or extracellular space. Macromolecular crowding can have different effect on amyloid formation as the excluded volume effect favours aggregation, whereas viscosity inhibits the kinetics of fibrilisation [32, 33, 35-37, 61]. We previously demonstrated under quiescent conditions, for $\mathrm{A} \beta$ a major key player in Alzheimer's disease pathogenesis, that the excluded volume effect dominates over viscosity in a crowder dose-dependent manner and also depending on the spatial heterogeneity of the system [36]. Moreover, we showed that the nature and properties of the crowding agent (dextran or Ficoll) also influence fibrilisation kinetics, recruitment to the AWI and morphology of the species formed. Similarly, but under non-quiescent conditions, Seeliger et al. showed that Ficoll and dextran also inhibit IAPP amyloidogenesis [37]. In this study, we investigated the effect of a different type of crowding agent, PVP, on IAPP amyloidogenesis under quiescent conditions. We used PVP as, in contrast to dextran and Ficoll, it permits the study of volume exclusion effects without the confounding effects of viscosity and surface activity, and also to study the relationship between polymer size and effects on assembly reactions.

IAPP fibrilisation was promoted by high molecular weight PVP360, which can be explained by the extent of the volume excluded by PVP360. Quantitation of the excluded volume for any flexible polymer in a solvent is complex because of the existence of several concentration-dependent regimes, and PVP360 is no exception. In a dilute regime, PVP360 chains remain dispersed and well separated from each other, so the volume occupancy can be easily calculated from the hydrodynamic radius, i.e. the concentration of individual chains is below the concentration where individual chains start to overlap. However, as the solution becomes more concentrated, the PVP360 chains will start to overlap, and the volume occupancy will cease to be linear $[62,63]$. The concentration at which this effect begins is the overlap concentration, $\mathrm{C}^{*}$, which can be calculated from the following equation[63]: $\mathrm{C}^{*}=$ $3 \mathrm{M} /\left(4 \pi \mathrm{N}_{\mathrm{A}} \mathrm{Rg}^{3}\right)$, in which $\mathrm{M}$ is the polymer molecular weight, $\mathrm{N}_{\mathrm{A}}$ is the Avogadro number and $\mathrm{R}_{\mathrm{g}}$ is the polymer radius of gyration (i.e. Stokes radius). PVP360 Stokes radius is $189.5 \AA$ [18]. For PVP360, we calculated $\mathrm{C}^{*}$ to be $20.97 \mathrm{~g} / \mathrm{L}$, which corresponds to a concentration of PVP360 of $58.25 \mu \mathrm{M}$ in a 100 $\mu \mathrm{l}$ reaction. The lowest concentration of PVP360 to significantly promote both elongation rate and plateau height is $5 \mu \mathrm{M}\left(3.011 \times 10^{14}\right.$ molecules in the $100 \mu \mathrm{l}$ reaction, with a Stokes radius of $\left.189.5 \AA\right)$, which is below $C^{*}$ and will occupy $8.58 \mu \mathrm{l}$ in a $100 \mu \mathrm{l}$ reaction $\left(3.011 \times 10^{14} \times 4 \pi / 3 \times(18.95)^{3}\right)$ [18]. However, the volume occupancy of PVP360 for the two highest concentrations ( 80 and $160 \mu \mathrm{M})$ could not be determined as they are above $\mathrm{C}^{*}$ and the volume occupancy ceases to be linear. Thus, for a given concentration, PVP360 would exclude a significant volume, which would confine IAPP in pockets of reduced volume within the PVP360 network (Figure 9A). Overall, this would lead to an increase in effective concentration of elongation competent IAPP monomers and thus promote elongation. As the PVP360 concentration increases, the volume fraction occupied by PVP360 increases accordingly, 
which confines IAPP in progressively smaller volumes therefore increasing even more the effective concentration.

No inhibition of IAPP assembly was observed for all PVP360 concentrations tested. This is in contrast to what was previously observed for the effect of the high molecular weight Ficoll400 on A $\beta$ fibrilogenesis [36]. At high Ficoll concentration (12\% w/v), inhibition of $A \beta$ assembly was due to both an increase in viscosity outweighing the excluded volume effect and competitive adsorption at the AWI between $A \beta$ and surface-active Ficoll reducing adsorption of every amyloid species [36]. 12\% Ficoll400 occupies $75 \mu \mathrm{l}$ of a $100 \mu \mathrm{l}$ solution, a similar volume occupancy would be achieved with $43.7 \mu \mathrm{M}$ PVP360, and the viscosities of the solutions would be $\sim 6.95$ and $2.93 \mathrm{cp}$ respectively (GE Healthcare Life Sciences) [36, 64]. 12\% Ficoll400 reduces the surface tension of water to $63 \mathrm{mN} / \mathrm{m}$, whereas 43.7 $\mu \mathrm{M}$ PVP360 only reduces it to $66.1 \mathrm{mN} / \mathrm{m}[36,64]$. Therefore, for a similar volume occupancy, Ficoll400 is 2.4 times more viscous than PVP360 and is also more surface active. Thus, PVP360 promotion of IAPP assembly is due to the beneficial excluded volume effect outweighing every other aspect.

In contrast, the lower molecular weight PVP10 inhibited IAPP assembly. The highest inhibitory concentration tested of PVP10 ( $80 \mu \mathrm{M}$, Stokes radius of $22 \AA)$ only occupied $0.21 \%$ of the total volume, which was too small volume occupancy to promote IAPP assembly via the excluded volume effect. The viscosity of $80 \mu \mathrm{M}$ PVP10 is only $2.84 \mathrm{cp}$ [19]. Therefore, PVP10 is less viscous than Ficoll400 (see above) and dextran40 (with a viscosity of $\sim 12.8 \mathrm{cp}$ for $12 \% \mathrm{w} / \mathrm{v}$ ) [36]. Thus, inhibition of IAPP elongation rate and plateau height by PVP10 is unlikely to be due to increased viscosity reducing diffusion rates. Murray et al. also showed that PVP either promoted or inhibited human insulin fibrilisation, with the effect depending on the PVP concentration and chain length [38]. The authors used different molecular weights of PVP to our study, PVP40 and PVP3.5. Nonetheless, they demonstrate that the effect of PVP on insulin fibrilisation scaled with the volume fraction occupied by PVP, as we observed with PVP10 and PVP360. The authors suggested that nucleation was not influenced by PVP induced limitations of diffusion but that elongation (i.e. fibril growth) was. Similarly in our study, at inhibitory concentrations, PVP10 preferentially impeded IAPP elongation rate rather than its nucleation rate.

Since IAPP inhibition by PVP10 could not be explained by a viscosity increase inhibiting the kinetics of fibrilisation, or the excluded volume effect promoting it, we investigated further PVP potential mode(s) of action. We examined whether PVP could interfere with IAPP recruitment and adsorption to the AWI. The dominant pathway to fibril formation would be that of the surface (AWI), rather than from bulk micelles, due to the catalytic effect of the AWI. PVP is unusual as it is highly water soluble, yet is amphiphilic (polar amide group and non-polar methyl groups) and exhibits weak surfactant properties. Indeed, PVP10, PVP40 and PVP360 were shown to decrease the surface tension of water (i.e. to adsorb to the AWI) but only marginally due to strong hydration of the PVP pyrrolidone ring $[64,65]$. We also found PVP10 to be weakly surface active and only for the first 10 min of our assay. Nonetheless and more importantly, PVP10 significantly reduced by $7.5 \%$ IAPP surface activity over our time course. It is possible that being weakly surface active, PVP10 interferes at the AWI with adsorption, orientation and alignment of a small proportion of IAPP monomers, processes necessary for elongation.

To determine further whether PVP10 affects assembly in the bulk solution and/or at the AWI, we also investigated the effect of a fixed PVP10 concentration across a range of IAPP concentrations spanning the CMC of IAPP in both the presence and absence of an AWI. In the presence of an AWI, for all concentrations tested, PVP10 inhibited the elongation rate (and this was significant for all except $1 \mu \mathrm{M}$ IAPP). The degree of inhibition decreasing as the IAPP concentration increased. In the absence of an AWI, PVP10 significantly inhibited both the elongation rate and plateau height, with the effect decreasing as the IAPP concentration increased. Moreover, the extent of the inhibition was bigger in absence of an AWI. Altogether our results strongly suggest that PVP10 preferentially inhibits IAPP elongation, that it is more active in the bulk solution than at the AWI, and that as IAPP concentration increases, the ratio of IAPP to PVP10 decreases, resulting in a 'dilution' of PVP10 inhibitory effect as fewer copies are present.

We propose the following model for PVP10 mode of action (Figure 9B, C and D). PVP10 acts in the bulk solution to block a small, but significant, proportion of IAPP monomers from reaching the 
AWI. It is possible that PVP does this by induced diffusion limitations, as was proposed for PVP and insulin [38]. However, it is more likely that by binding to IAPP, PVP sequesters monomers in the bulk solution in an elongation-incompetent form. PVP has previously been shown to be able to form weak soft interactions with protein native state and here we clearly demonstrate that IAPP selectively interacts with PVP10 with a strong binding affinity [66].

Moreover, it is also possible that by being weakly surface active, PVP10 also depletes the AWI from a proportion of adsorbed IAPP monomers and sequesters them in an adsorption/elongation incompetent form. Our rationale for proposing that PVP10 interacts with IAPP monomers comes from several lines of evidence: PVP10 systematic inhibition of IAPP elongation, which relies on monomer addition to nuclei and/or protofibrils; PVP10 inhibition of IAPP plateau, which again relies on available monomers, and not on micelles or other assembly species, to extend protofibrils and fibrils; PVP10 is only surface active for the first $10 \mathrm{~min}$ and still able to significantly reduce IAPP surface activity, a time frame during an assembly reaction only relating to IAPP monomers; PVP10 inhibitory effect on IAPP elongation is higher in absence of an AWI than in the presence of an AWI (2.37 versus 1.34 fold for 4 $\mu \mathrm{M}$, and 1.36 versus 1.21 fold for $12 \mu \mathrm{M}$ ), due to more PVP10 copies present in the bulk to sequester more monomers; and finally, our MST data were carried out immediately after mixing, i.e. $t=0$, therefore PVP10 binding is most likely to occur to IAPP monomers. Below the CMC, the only assembly pathway available to IAPP is the AWI, so overall, IAPP assembly is inhibited. Above the CMC, IAPP can assemble both in the bulk and at the AWI. The AWI pathway is still partially inhibited by PVP10 and now the bulk solution pathway is also inhibited by sequestering monomer in an elongation incompetent form. As IAPP concentration increases, the PVP chains become saturated with interacting IAPP monomers, allowing enough IAPP elongation competent monomers to remain free in the solution to elongate some nuclei, resulting in a lesser inhibitory effect. In absence of an AWI, more PVP10 copies are available in the bulk solution, as not adsorbed to the AWI, to sequester more monomers in an elongation incompetent form. Overall, this affects both elongation and the final amount of fibrils produced, and the inhibitory effect is more pronounced than in presence of an AWI.

A biphasic behaviour was observed during IAPP elongation, mostly in presence of PVP (both PVP10 and PVP360) (Figure 2, 3, S1, and S2). To calculate IAPP elongation rate, we selected the slope of the second inflection point. The inflection points were determined as the time-point at which the angle between tangent fits of the straight line before and after that time-point showed the greatest value. The first phase defined in this way showed no dependence upon conditions (including PVP10 or PVP360 concentration) suggesting that this process is not susceptible to promotion or inhibition. However, the second phase showed clear dependence upon experimental conditions, suggesting that a different underlying process has supervened that is susceptible to modification by either polymer length of PVP. Similar biphasic behaviour has been previously seen for IAPP alone, or in presence of glycosaminoglycans [67-69]. In the case of IAPP, or a mixture of IAPP and I 26 P-IAPP, the biphasic behaviour was thought to be due to the formation of glycosaminoglycan-bound intermediates $[67,68]$. A biphasic growth curve was also observed for the fibrilisation of lysozyme in presence of mature fibrils [70]. More interestingly, a stepwise increase in the ThT intensity was observed for insulin B chain peptide, with an initial increase followed by another increase before reaching a plateau [71]. This twophase increase was reproducible and shown to be due to the transient formation of on-pathway metastable prefibrilar aggregate intermediates (first phase) followed by fibril formation (second phase). The authors hypothesised that the intermediates can serve as pre-nucleation clusters favouring nucleation by increasing the effective local concentration of monomers, intermolecular interactions, and the hydrophobic environment. Here, we have not investigated further the cause of IAPP biphasic behaviour, which could be the subject of further studies. However, one could speculate that the first increase of IAPP two-phase elongation might be due to the formation of prefibrillar intermediates, as seen for insulin B chain peptide, which might be either enhanced or stabilised by PVP. PVP360 could promote these via the excluded volume effect, i.e. by increasing the effective monomer concentration for intermediate formation, whereas PVP10 could stabilise these via binding.

In this study, we demonstrate that the threshold between promotion of IAPP fibrilisation and inhibition during macromolecular crowding by PVP is dependent on the chain length of PVP and the effect associated with the presence of the polymer. IAPP fibrilisation was promoted by high molecular weight PVP360 solely due to beneficial volume exclusion overweighting any weak increase in viscosity and weak surfactant properties. In contrast, inhibition of IAPP fibrilisation by low molecular weight 
PVP10 could not be explained by a significant increase in solution viscosity but could by PVP-induced reduction of IAPP adsorption to the AWI and direct PVP10-IAPP interaction sequestering monomers in an elongation incompetent form. Overall, the inhibitory effect was more prominent in the bulk solution. Thus, we clearly show that PVP10 does not behave like other model crowding agents such as Ficoll or dextran, and has unique inhibitory properties. Therefore, the use of different types of crowder polymers, and/or different volume fraction and chain length of the same crowder polymer is necessary when investigating in vitro the effect of macromolecular crowding on any system, and in particular amyloid formation. Physiologically, the in vivo crowded environment of the cell or of the extracellular space would affect the amyloid assembly process and its pathogenic consequences. In our modern societies, there is a rapidly growing number of people affected by amyloid diseases, for which there are currently no cures. The development of disease-modifying drugs has been so far unsuccessful, partly due to our still limited mechanistic understanding of these diseases, but also due to our limited understanding of amyloid interactions with drugs or small molecules. A range of strategies have been used to identify therapeutic compounds, one of which focusses on inhibiting or modulating aggregation in various ways (e.g. inhibiting toxic oligomer formation or promoting their degradation, stabilising non-toxic species). Screening for small molecules in in-vitro and cell-based assays remain the main approaches currently used. Thus, the finding of a short polymer with unique inhibitory properties on amyloid assembly could serve as a stepping stone in the discovery of novel therapeutic agents to target amyloidogenesis. Indeed, small molecules could be designed around the PVP chemical entity/backbone, and screened for their ability to inhibit the formation of toxic species by binding to monomers and sequestering them in an elongation incompetent form.

Acknowledgements: We are grateful to Susan Lea, Andreas Haensele and Stephen Johnson (Dunn School of Pathology, Oxford) for help with the microscale thermophoresis experiments. We would also like to thank Omer Dushek (Dunn School of Pathology, Oxford) for his help with analysis of MST data using GraphPad Prism.

Competing interests: The authors declare that they have no conflicts of interest with the contents of this article.

Funding: LJ was supported by a research grant from Synaptica Ltd.

Author contributions: LJ and DJV designed the study. LJ and RB performed the experiments. LJ and $\mathrm{RB}$ performed the analysis and LJ led the writing of the paper. LJ and DJV discussed results, interpreted the data and commented on the manuscript.

\section{References}




\section{FIGURE LEGENDS}
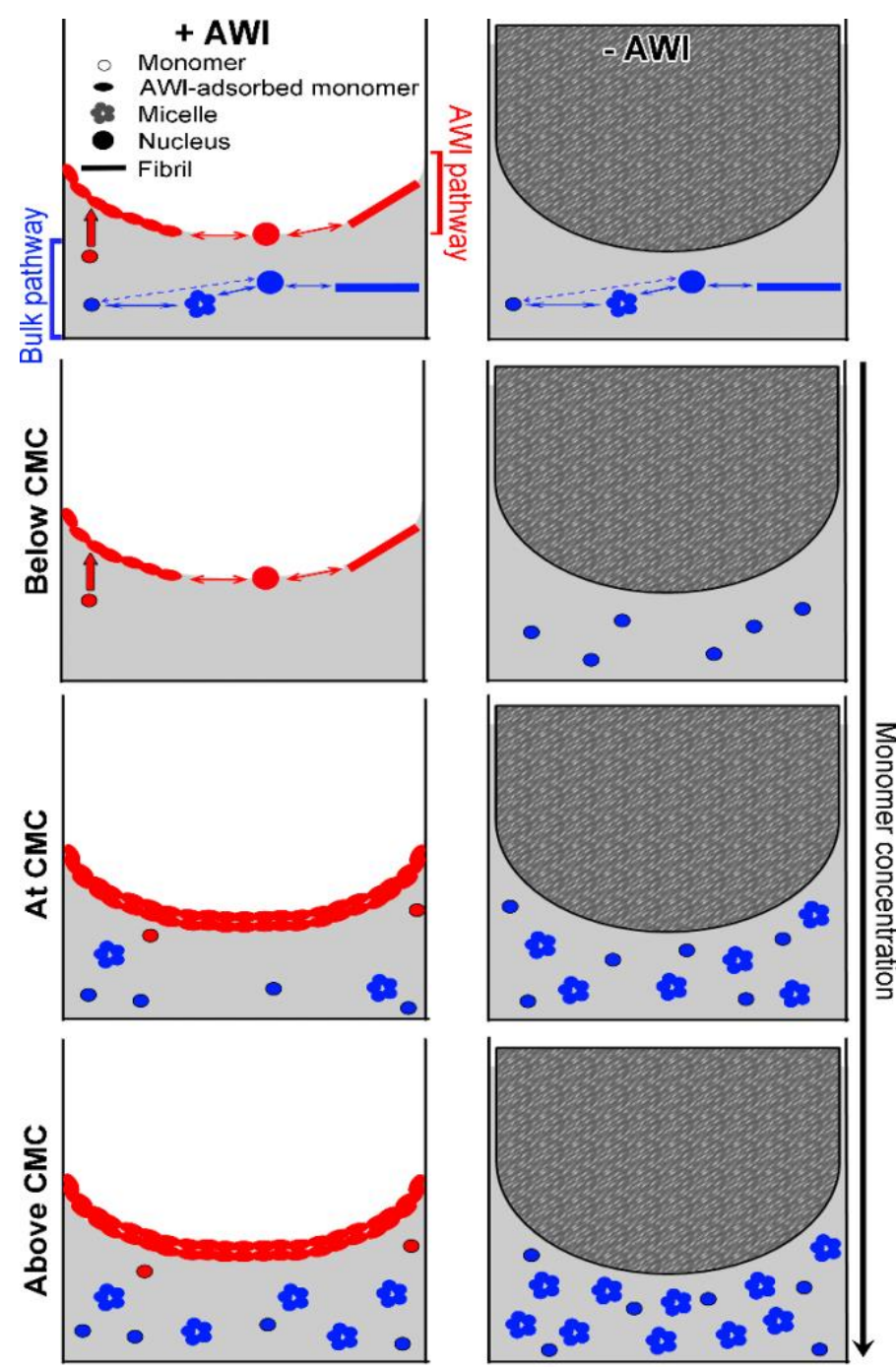

Figure 1. Schematic representation of the surface and bulk pathways of amyloidogenesis.

Being surfactants, amyloid monomers adsorb to HHIs (such as the AWI), which catalyses amyloid formation. From these adsorbed monomers, nuclei will form and subsequently fibrils, which will remain at the AWI. The interfacial volume at the AWI is smaller than the bulk volume (a 2D versus a 3D system), which increases the effective monomer concentration and allows nucleation to occur more rapidly than in the bulk solution. In the bulk solution, nucleation will only occur when the monomer concentration is at or above the CMC and when the AWI is saturated with adsorbed peptides. Thus, in presence of an AWI, the majority of nucleation and elongation will occur rapidly at the AWI (left panels). In contrast, in absence of an AWI, the slow bulk pathway would be the only nucleation pathway and would rely on micelle formation when the monomer concentration is at or above the CMC (right panels). Increasing the concentration of monomers above the CMC only allows the formation of more micelles in the bulk solution (thus increasing the bulk nucleation rate) since the AWI, if present, is already fully saturated with monomers and the surface pathway is at its optimum and dominates. 

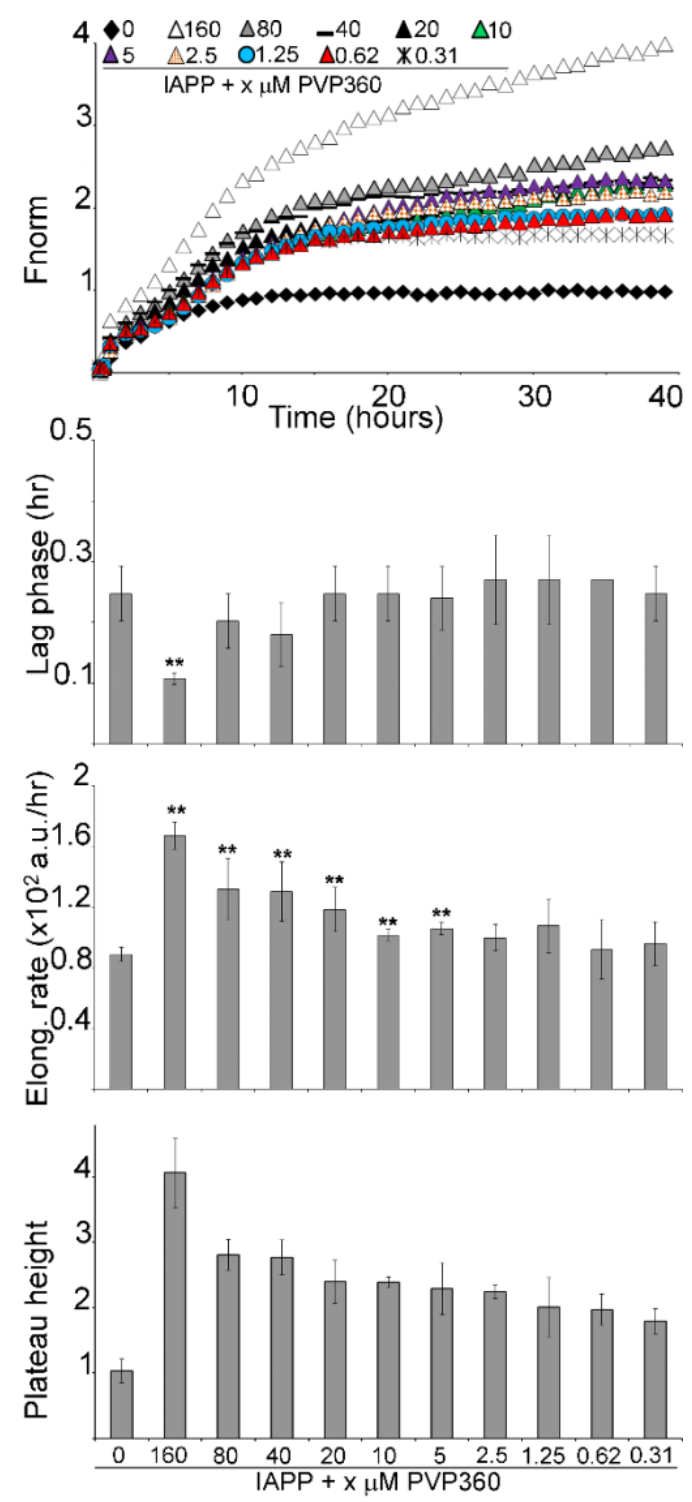

Figure 2: PVP360 promotes IAPP fibrilisation.

$1.82 \mu \mathrm{M}$ IAPP, in $32 \mu \mathrm{M}$ ThT and PBS, was incubated under non-agitating conditions in absence or presence of varying concentrations of PVP360. Changes in ThT fluorescence were monitored (top panel), with the lag phase, elongation rate and plateau height (bottom panels) depicted. ' $* *$ ' $P<0.03$ when compared to IAPP without PVP360. The mean of at least three independent assays is shown. Error bars represent \pm standard error of the mean. Fnorm: normalised fluorescence, a.u.: arbitrary units. 

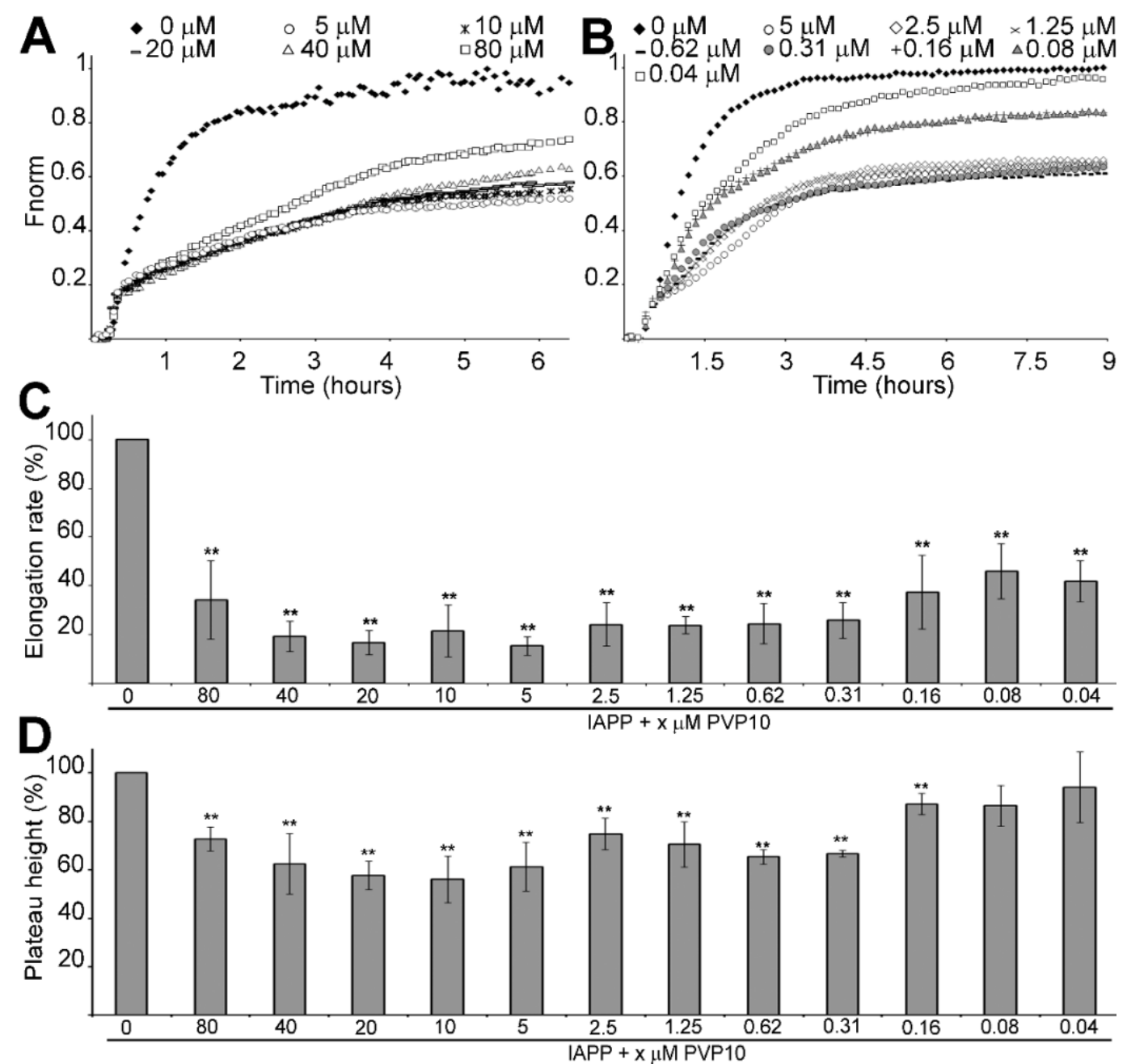

Figure 3: PVP10 inhibits IAPP fibrilisation.

$1.82 \mu \mathrm{M}$ IAPP, in $32 \mu \mathrm{M}$ ThT and PBS, was incubated under non-agitating conditions in absence or presence of varying concentrations of PVP10, ranging from 0.04 to $80 \mu \mathrm{M}$. Changes in ThT fluorescence were monitored (A and $\mathbf{B})$. Percentage changes for IAPP elongation rate $(\mathbf{C})$ and plateau height (D) in presence of PVP10, when compared to IAPP in absence of PVP10, are depicted. '**, $P<0.039$ when compared to IAPP without PVP10. The mean of at least three independent assays is shown. Error bars represent \pm standard error of the mean. Fnorm: normalised fluorescence, a.u.: arbitrary units. 


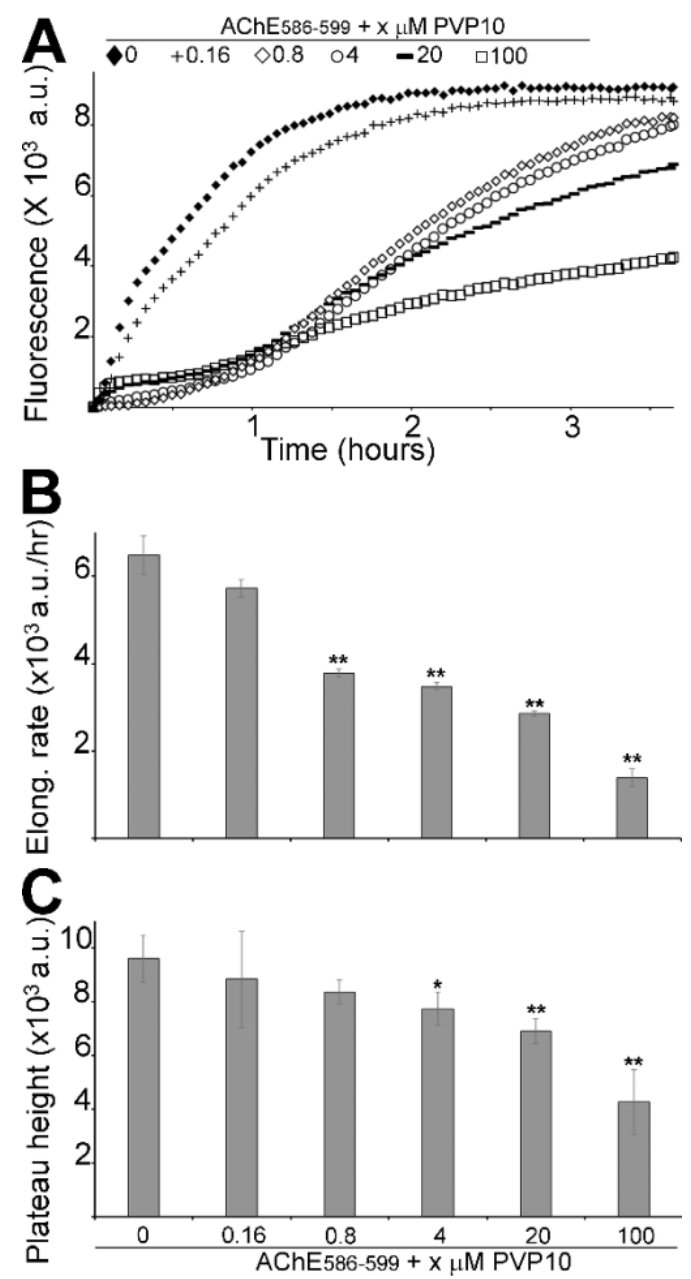

Figure 4: PVP10 inhibits $\mathrm{AChE}_{586-599}$ fibrilisation.

$125 \mu \mathrm{M}$ AChE $586-599$, in $165 \mu \mathrm{M}$ ThT and PBS, was incubated under agitating conditions in absence or presence of varying concentrations of PVP10, ranging from 0.16 to $100 \mu \mathrm{M}$. Changes in ThT fluorescence were monitored (A), with the lag phase, elongation rate and plateau height (B and $\mathbf{C})$ depicted. '*' $P<0.05$ and '**' $P<0.03$ when compared to $\mathrm{AChE}_{586-599}$ without PVP10. The mean of at least three independent assays is shown. Error bars represent \pm standard error of the mean. a.u.: arbitrary units.

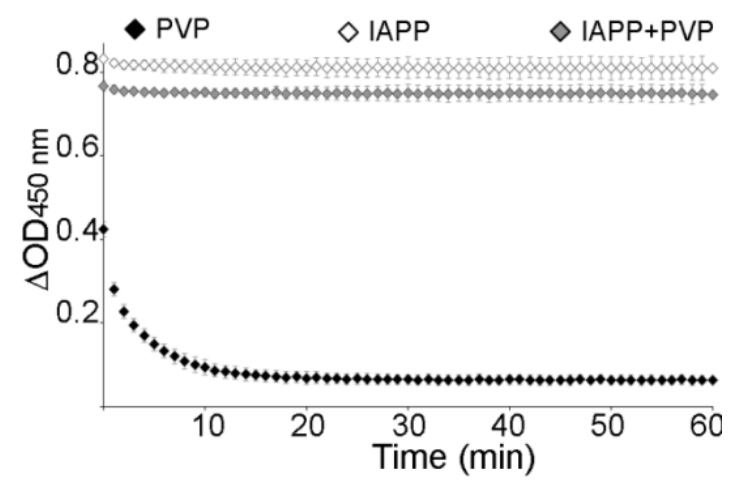

Figure 5: IAPP adsorption to the AWI is reduced by PVP10.

The surface activity for a solution of $1.2 \mu \mathrm{M}$ IAPP, in absence or presence of $5 \mu \mathrm{M}$ PVP10, was monitored over time. $\triangle \mathrm{OD}$ calculations were as described in Experimental. a.u.: arbitrary units. The mean of at least three independent assays is shown. Error bars represent \pm standard error of the mean. 


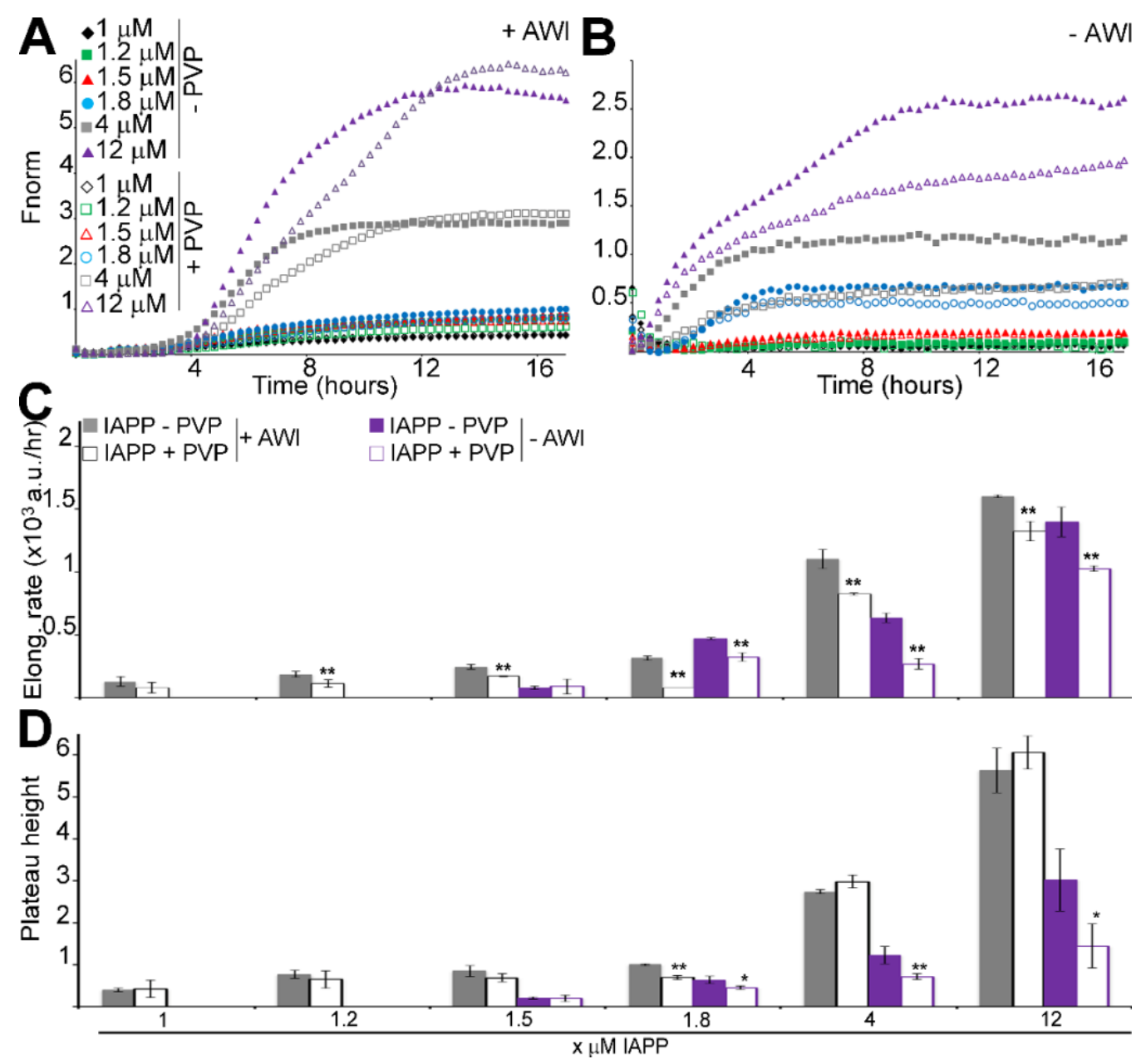

Figure 6: The effect of PVP10 on IAPP fibrilisation, in absence or presence of an AWI.

Several concentrations of IAPP, in $32 \mu \mathrm{M}$ ThT and PBS, were incubated under non-agitating conditions in absence or presence of $5 \mu \mathrm{M}$ PVP10. Changes in ThT fluorescence were monitored in presence (A) and absence of an AWI (B). IAPP elongation rate $(\mathbf{C})$ and plateau height $(\mathbf{D})$ in presence of PVP10, when compared to IAPP in absence of PVP10, are depicted. ' $*$ ' $P<0.05$ and ' $* *$ ' $P<0.03$ when compared to IAPP without PVP10. The mean of at least three independent assays is shown. Error bars represent \pm standard error of the mean. Fnorm: normalised fluorescence, a.u.: arbitrary units, elong. rate: elongation rate. 


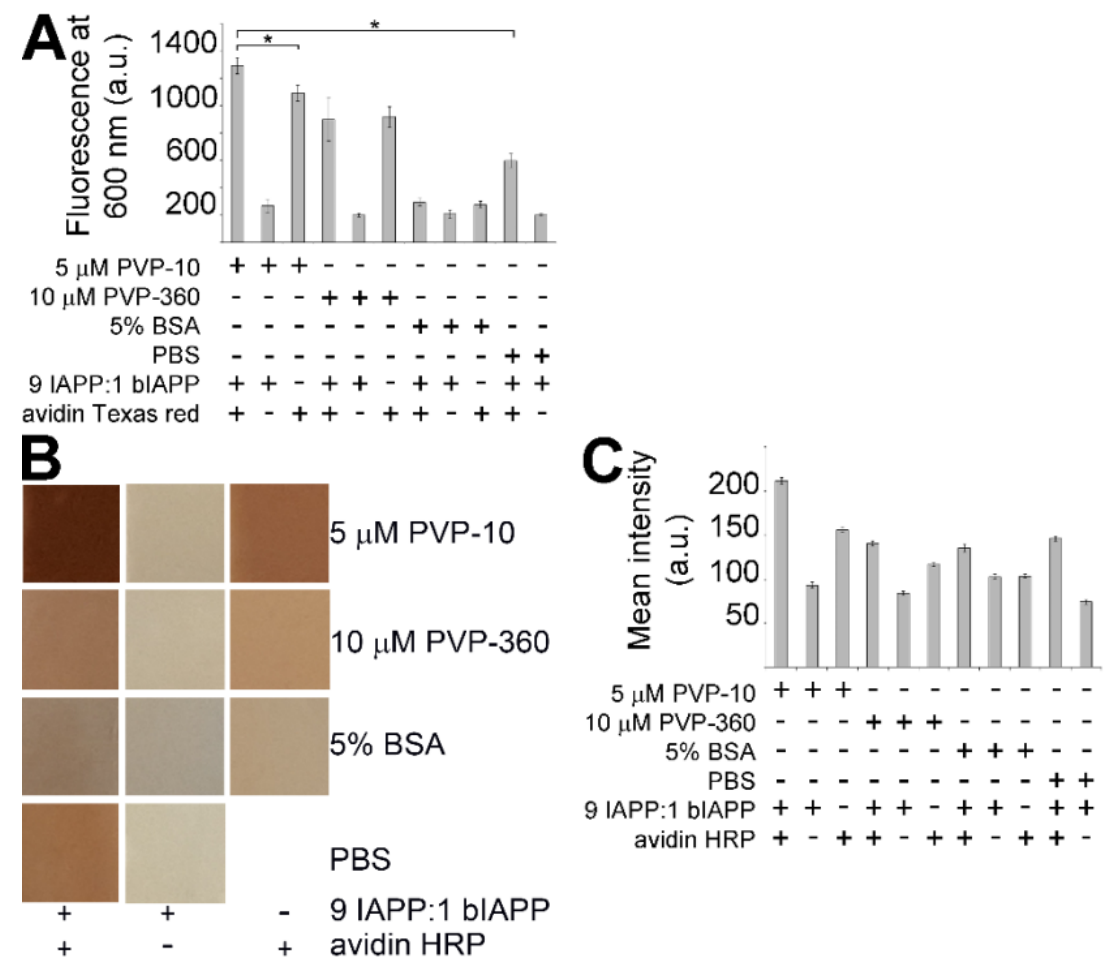

Figure 7: PVP10 interacts with IAPP.

Wells of a 96-well plate (A) or strips of nitrocellulose (B) were incubated in PBS with $5 \mu$ M PVP10, or $10 \mu \mathrm{M}$ PVP360, or 5\% BSA, or no polymer overnight. The wells or nitrocellulose strips were then incubated with $3.6 \mu \mathrm{M}$ IAPP and $0.4 \mu \mathrm{M}$ bIAPP, or PBS, for $5 \mathrm{~h}$ at room temperature, before incubation with avidin Texas red (A) or avidin HRP (B), or PBS for $30 \mathrm{~min}$. (A) Texas red fluorescence was measured and shown are the test wells with controls subtracted (no IAPP control, and no avidin control). The mean of at least three independent assays is shown. ' $*$ ' $P<0.008$. a.u.: arbitrary units. Error bars represent \pm standard error of the mean. (B) HRP activity was detected by using DAB. (C) The intensity of the DAB colouration was quantified by image analysis in FIJI and is presented as a mean intensity. a.u.: arbitrary units. Error bars represent \pm standard error of the mean of the quantitation of the DAB colouration from each nitrocellulose strips. 


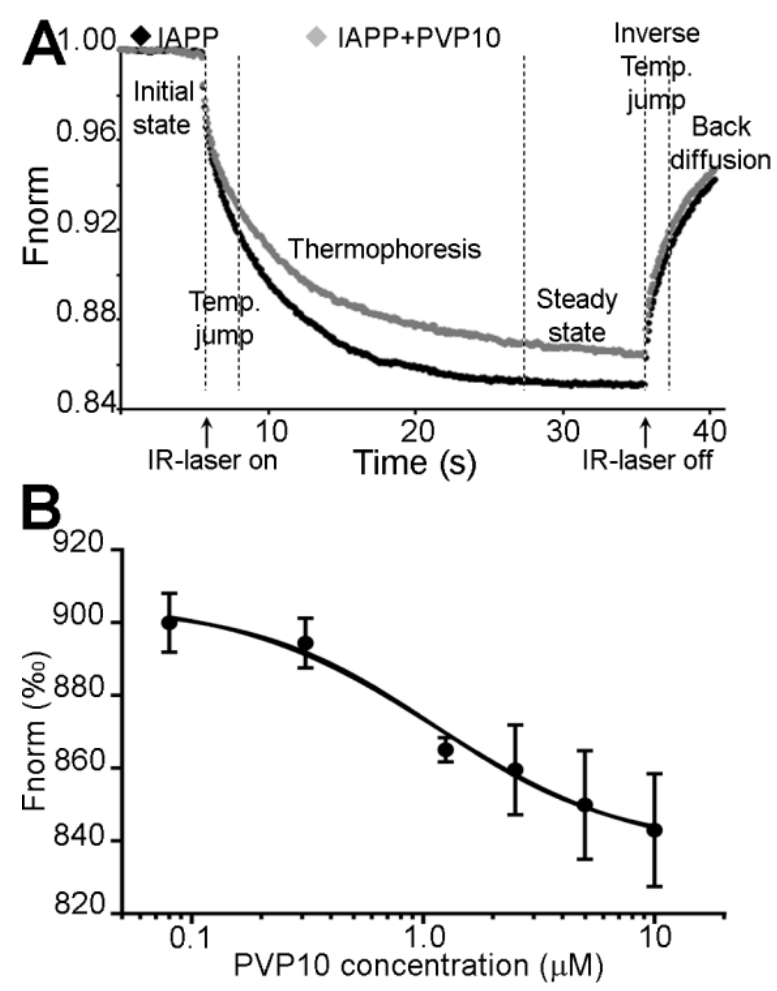

Figure 8. PVP10-IAPP interaction by microscale thermophoresis.

(A) Shown are normalised fluorescence time traces of 3.6 $\mu \mathrm{M}$ IAPP, $0.4 \mu \mathrm{M}$ bIAPP, $0.08 \mu \mathrm{M}$ avidin fluorescein in PBS, in absence or presence of $0.08 \mu \mathrm{M}$ PVP10. Measurement were made at $\mathrm{t}=0$, immediately after mixing and at $25^{\circ} \mathrm{C}$. A series of processes can be separated from each other: initial state, temperature (temp.) jump, thermophoresis, steady state, inverse temperature jump and back diffusion. The mean of at least three independent assays is shown. (B) Kd determination. Binding curve of PVP10 to IAPP. 3.6 $\mu \mathrm{M}$ IAPP, $0.4 \mu \mathrm{M}$ bIAPP, $0.08 \mu \mathrm{M}$ avidin fluorescein in PBS were mixed with increasing concentrations of PVP10. Measurement were made at $\mathrm{t}=0$, immediately after mixing and at $25^{\circ} \mathrm{C}$. The fitting is based on thermophoresis $+\mathrm{T}$ jump data. The mean of at least three independent assays is shown. Error bars represent \pm standard error of the mean. 

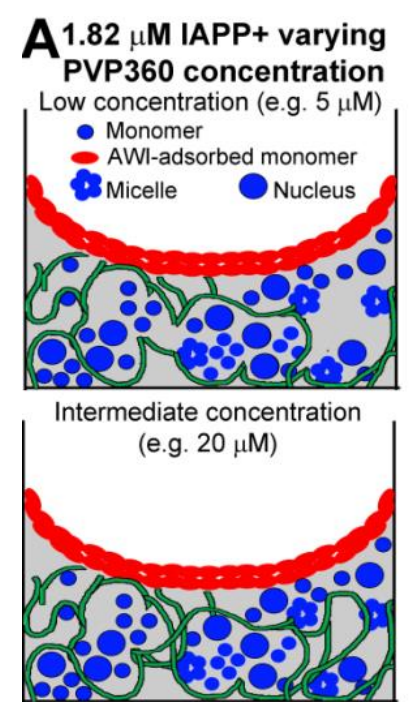

High concentration

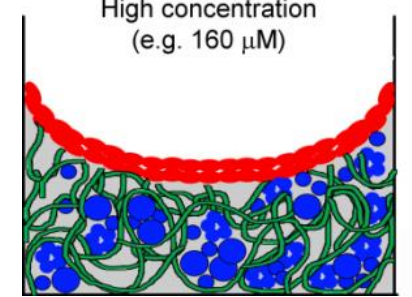

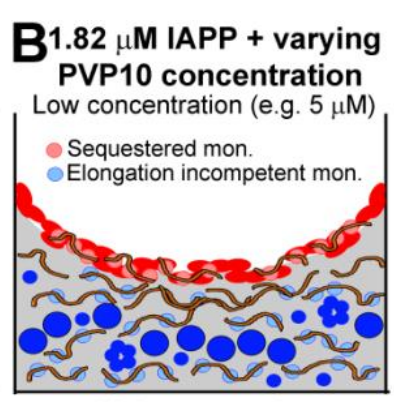

High concentration

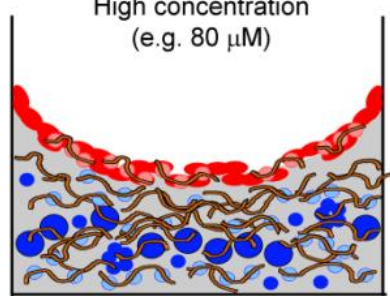

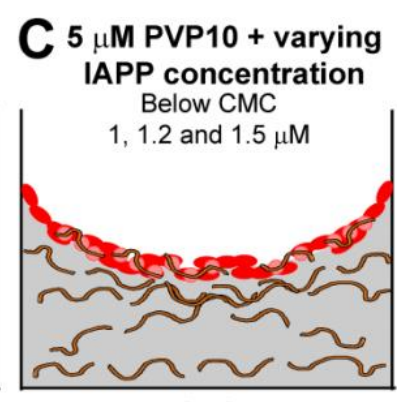

Above CMC ( $4 \mu \mathrm{M})$

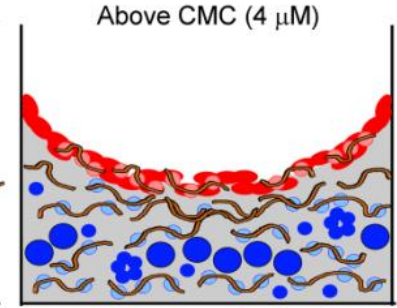

Above CMC (12 $\mu \mathrm{M})$

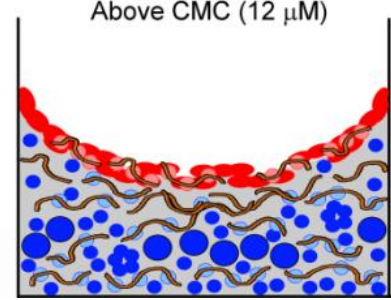

D $5 \mu \mathrm{M}$ PVP10 + varying IAPP concentration

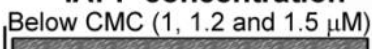

Figure 9: Model for PVP mode of action on IAPP assembly.

(A) PVP360 promotes IAPP assembly. As PVP360 concentration increases (from top to bottom panel), the volume excluded also increases, which confines IAPP in progressively smaller volumes therefore increasing even more the effective concentration of elongation-competent monomers. (B) A fixed IAPP concentration, just above the CMC, in the presence of increasing PVP10 concentrations. Surface active PVP10 not only depletes the AWI of some adsorbed IAPP monomers, but also interacts with monomers to sequester them in an adsorption/elongation-incompetent form. Thus, the AWI pathway is inhibited to some extend by PVP10. In the bulk solution, PVP10 also interacts with IAPP monomers, which renders them elongation incompetent. Overall, inhibition of elongation is observed. 'mon.' stands for monomer. (C) A fixed PVP concentration, $5 \mu \mathrm{M}$, in presence of increasing concentration of IAPP and in presence of an AWI. (Top panel) Below the CMC, IAPP assembly at the AWI is fully inhibited by PVP10 as it is the only assembly pathway available and surface active PVP10 depletes some adsorbed IAPP monomers from it and sequesters them in an adsorption/elongation incompetent form. (Middle panel) Above the CMC, IAPP can assemble both in the bulk and at the AWI, and both pathways are partially inhibited. In the bulk solution, PVP10 chains are now saturated with interacting IAPP monomers ( 2.2 fold more than in the top panel), allowing enough elongation competent monomers to remain free in the solution to elongate some nuclei, resulting in a lesser inhibitory effect than that seen in (B, top panel). (Bottom panels) As IAPP concentration increases even further above the CMC, and as PVP chains are already saturated, the amount of elongation competent monomers remaining in the solution further increases, resulting in an even smaller inhibitory effect. (D) A fixed PVP concentration, $5 \mu \mathrm{M}$, in presence of increasing concentration of IAPP and in absence of an AWI. (Top panel) Below the CMC, assembly is completely inhibited as the AWI has been removed and PVP10 sequesters monomers in the bulk in an elongation-incompetent form. (Middle and bottom panels) Above the CMC, IAPP micelle formation in the bulk is inhibited by PVP10, even further than in presence of an AWI. Without the AWI, more PVP10 copies are available in the bulk solution, as they are not adsorbed to an AWI, to sequester more monomers in an elongation-incompetent form. Overall, this affects both elongation and also the final amount of fibrils produced. 
1. Stefani, M. and Dobson, C.M. (2003) Protein aggregation and aggregate toxicity: new insights into protein folding, misfolding diseases and biological evolution. $J$ Mol Med (Berl). 81, 678-699.

2. Chiti, F. and Dobson, C.M. (2006) Protein misfolding, functional amyloid, and human disease. Annu Rev Biochem. 75, 333-366.

3. Gazit, E. (2002) A possible role for pi-stacking in the self-assembly of amyloid fibrils. Faseb J. 16, 77-83.

4. Abedini, A. and Schmidt, A.M. (2013) Mechanisms of islet amyloidosis toxicity in type 2 diabetes. Febs Letters. 587, 1119-1127.

5. Westermark, P., Wernstedt, C., Wilander, E., Hayden, D.W., Obrien, T.D. and Johnson, K.H. (1987) Amyloid Fibrils in Human Insulinoma and Islets of Langerhans of the Diabetic Cat Are Derived from a Neuropeptide-Like Protein Also Present in Normal Islet Cells. P Natl Acad Sci USA. 84, 3881-3885.

6. Lutz, T.A. (2005) Pancreatic amylin as a centrally acting satiating hormone. Curr Drug Targets. 6, 181-189.

7. Hoppener, J.W.M. and Lips, C.J.M. (2006) Role of islet amyloid in type 2 diabetes mellitus. Int J Biochem Cell B. 38, 726-736.

8. Jayasinghe, S.A. and Langen, R. (2007) Membrane interaction of islet amyloid polypeptide. Bba-Biomembranes. 1768, 2002-2009.

9. Lomakin, A., Teplow, D.B., Kirschner, D.A. and Benedek, G.B. (1997) Kinetic theory of fibrillogenesis of amyloid beta-protein. P Natl Acad Sci USA. 94, 7942-7947.

10. Soreghan, B., Kosmoski, J. and Glabe, C. (1994) Surfactant properties of Alzheimer's A beta peptides and the mechanism of amyloid aggregation. J Biol Chem. 269, 28551-28554.

11. Cottingham, M.G., Bain, C.D. and Vaux, D.J. (2004) Rapid method for measurement of surface tension in multiwell plates. Lab. Invest. 84, 523-529.

12. Lopes, D.H., Meister, A., Gohlke, A., Hauser, A., Blume, A. and Winter, R. (2007) Mechanism of islet amyloid polypeptide fibrillation at lipid interfaces studied by infrared reflection absorption spectroscopy. Biophys J. 93, 3132-3141.

13. Parhi, P., Golas, A., Barnthip, N., Noh, H. and Vogler, E.A. (2009) Volumetric interpretation of protein adsorption: capacity scaling with adsorbate molecular weight and adsorbent surface energy. Biomaterials. 30, 6814-6824.

14. Krishnan, A., Siedlecki, C.A. and Vogler, E.A. (2003) Traube-rule interpretation of protein adsorption at the liquid-vapor interface. Langmuir. 19, 10342-10352.

15. Terzi, E., Holzemann, G. and Seelig, J. (1997) Interaction of Alzheimer beta-amyloid peptide(1-40) with lipid membranes. Biochemistry. 36, 14845-14852.

16. Jean, L., Lee, C.F., Lee, C., Shaw, M. and Vaux, D.J. (2010) Competing discrete interfacial effects are critical for amyloidogenesis. FASEB J. 24, 309-317.

17. Jean, L., Lee, C.F. and Vaux, D.J. (2012) Enrichment of amyloidogenesis at an airwater interface. Biophys J. 102, 1154-1162.

18. Armstrong, J.K., Wenby, R.B., Meiselman, H.J. and Fisher, T.C. (2004) The hydrodynamic radii of macromolecules and their effect on red blood cell aggregation. Biophysical Journal. 87, 4259-4270.

19. Qiu, P.H. and Mao, C.B. (2011) Viscosity Gradient as a Novel Mechanism for the Centrifugation-Based Separation of Nanoparticles. Adv Mater. 23, 4880-4885.

20. Kayed, R., Sokolov, Y., Edmonds, B., McIntire, T.M., Milton, S.C., Hall, J.E. and Glabe, C.G. (2004) Permeabilization of lipid bilayers is a common conformationdependent activity of soluble amyloid oligomers in protein misfolding diseases. Journal of Biological Chemistry. 279, 46363-46366. 
21. Brangwynne, C.P., Mitchison, T.J. and Hyman, A.A. (2011) Active liquid-like behavior of nucleoli determines their size and shape in Xenopus laevis oocytes. Proceedings of the National Academy of Sciences. 108, 4334-4339.

22. Kreuger, J., Spillmann, D., Li, J.-p. and Lindahl, U. (2006) Interactions between heparan sulfate and proteins: the concept of specificity. The Journal of Cell Biology. 174, 323-327.

23. Engel, M.F.M., Khemtemourian, L., Kleijer, C.C., Meeldijk, H.J.D., Jacobs, J., Verkleij, A.J. et al. (2008) Membrane damage by human islet amyloid polypeptide through fibril growth at the membrane. P Natl Acad Sci USA. 105, 6033-6038.

24. Demuro, A., Mina, E., Kayed, R., Milton, S.C., Parker, I. and Glabe, C.G. (2005) Calcium dysregulation and membrane disruption as a ubiquitous neurotoxic mechanism of soluble amyloid oligomers. Journal of Biological Chemistry. 280, 17294-17300.

25. Wang, X., Permentier, H.P., Rink, R., Kruijtzer, J.A.W., Liskamp, R.M.J., Wösten, H.A.B. et al. (2004) Probing the self-assembly and the accompanying structural changes of hydrophobin SC3 on a hydrophobic surface by mass spectrometry. Biophysical journal. 87, 1919-1928.

26. Linder, M.B. (2009) Hydrophobins: Proteins that self assemble at interfaces. Current Opinion in Colloid \& Interface Science. 14, 356-363.

27. Fulton, A.B. (1982) How crowded is the cytoplasm? Cell. 30, 345-347.

28. Ellis, R.J. (2001) Macromolecular crowding: an important but neglected aspect of the intracellular environment. Curr Opin Struct Biol. 11, 114-119.

29. Minton, A.P. (2000) Effect of a concentrated "inert" macromolecular cosolute on the stability of a globular protein with respect to denaturation by heat and by chaotropes: a statistical-thermodynamic model. Biophys J. 78, 101-109.

30. Minton, A.P. (2000) Implications of macromolecular crowding for protein assembly. Curr Opin Struct Biol. 10, 34-39.

31. Zhou, H.X., Rivas, G. and Minton, A.P. (2008) Macromolecular crowding and confinement: biochemical, biophysical, and potential physiological consequences. Annu Rev Biophys. 37, 375-397.

32. Munishkina, L.A., Cooper, E.M., Uversky, V.N. and Fink, A.L. (2004) The effect of macromolecular crowding on protein aggregation and amyloid fibril formation. $J$ Mol Recognit. 17, 456-464.

33. Uversky, V.N., E, M.C., Bower, K.S., Li, J. and Fink, A.L. (2002) Accelerated alphasynuclein fibrillation in crowded milieu. FEBS Lett. 515, 99-103.

34. Hatters, D.M., Minton, A.P. and Howlett, G.J. (2002) Macromolecular crowding accelerates amyloid formation by human apolipoprotein C-II. J Biol Chem. 277, 7824-7830.

35. Zhou, Z., Fan, J.B., Zhu, H.L., Shewmaker, F., Yan, X., Chen, X. et al. (2009) Crowded cell-like environment accelerates the nucleation step of amyloidogenic protein misfolding. J Biol Chem. 284, 30148-30158.

36. Lee, C.F., Bird, S., Shaw, M., Jean, L. and Vaux, D.J. (2012) Combined effects of agitation, macromolecular crowding, and interfaces on amyloidogenesis. J Biol Chem. 287, 38006-38019.

37. Seeliger, J., Werkmuller, A. and Winter, R. (2013) Macromolecular Crowding as a Suppressor of Human IAPP Fibril Formation and Cytotoxicity. Plos One. 8.

38. Murray, B., Rosenthal, J., Zheng, Z.L., Isaacson, D., Zhu, Y.X. and Belfort, G. (2015) Cosolute Effects on Amyloid Aggregation in a Nondiffusion Limited Regime: 
Intrinsic Osmolyte Properties and the Volume Exclusion Principle. Langmuir. 31, 4246-4254.

39. Ravin, H.A., Seligman, A.M. and Fine, J. (1952) Polyvinyl Pyrrolidone as a Plasma Expander - Studies on Its Excretion, Distribution and Metabolism. New Engl J Med. 247, 921-929.

40. Cottingham, M.G., Voskuil, J.L. and Vaux, D.J. (2003) The intact human acetylcholinesterase C-terminal oligomerization domain is alpha-helical in situ and in isolation, but a shorter fragment forms beta-sheet-rich amyloid fibrils and protofibrillar oligomers. Biochemistry. 42, 10863-10873.

41. Shen, C.L. and Murphy, R.M. (1995) Solvent effects on self-assembly of betaamyloid peptide. Biophys J. 69, 640-651.

42. Arosio, P., Knowles, T.P. and Linse, S. (2015) On the lag phase in amyloid fibril formation. Phys Chem Chem Phys. 17, 7606-7618.

43. Bentea, L., Watzky, M.A. and Finke, R.G. (2017) Sigmoidal Nucleation and Growth Curves Across Nature Fit by the Finke-Watzky Model of Slow Continuous Nucleation and Autocatalytic Growth: Explicit Formulas for the Lag and Growth Times Plus Other Key Insights. J Phys Chem C. 121, 5302-5312.

44. Cameron, A.M. (1954) Blood plasma expander-PVP. Del Med J. 26, 149-151.

45. Altman, G.H., Diaz, F., Jakuba, C., Calabro, T., Horan, R.L., Chen, J. et al. (2003) Silkbased biomaterials. Biomaterials. 24, 401-416.

46. LeVine, H., 3rd (1993) Thioflavine $\mathrm{T}$ interaction with synthetic Alzheimer's disease beta-amyloid peptides: detection of amyloid aggregation in solution. Protein Sci. 2, 404-410.

47. Cottingham, M.G., Hollinshead, M.S. and Vaux, D.J. (2002) Amyloid fibril formation by a synthetic peptide from a region of human acetylcholinesterase that is homologous to the Alzheimer's amyloid-beta peptide. Biochemistry. 41, 1353913547.

48. Bohrmann, B., Tjernberg, L., Kuner, P., Poli, S., Levet-Trafit, B., Naslund, J. et al. (1999) Endogenous proteins controlling amyloid beta-peptide polymerization. Possible implications for beta-amyloid formation in the central nervous system and in peripheral tissues. J Biol Chem. 274, 15990-15995.

49. Luo, J., Warmlander, S.K., Graslund, A. and Abrahams, J.P. (2014) Non-chaperone proteins can inhibit aggregation and cytotoxicity of Alzheimer amyloid beta peptide. J Biol Chem. 289, 27766-27775.

50. Finn, T.E., Nunez, A.C., Sunde, M. and Easterbrook-Smith, S.B. (2012) Serum albumin prevents protein aggregation and amyloid formation and retains chaperone-like activity in the presence of physiological ligands. J Biol Chem. 287, 21530-21540.

51. Algamal, M., Ahmed, R., Jafari, N., Ahsan, B., Ortega, J. and Melacini, G. (2017) Atomic-resolution map of the interactions between an amyloid inhibitor protein and amyloid beta (Abeta) peptides in the monomer and protofibril states. J Biol Chem. 292, 17158-17168.

52. Duhr, S. and Braun, D. (2006) Thermophoretic depletion follows Boltzmann distribution. Phys Rev Lett. 96, 168301.

53. Duhr, S. and Braun, D. (2006) Why molecules move along a temperature gradient. Proc Natl Acad Sci U S A. 103, 19678-19682.

54. Jerabek-Willemsen, M., Andre, T., Wanner, R., Roth, H.M., Duhr, S., Baaske, P. and Breitsprecher, D. (2014) MicroScale Thermophoresis: Interaction analysis and beyond. Journal of Molecular Structure. 1077, 101-113. 
55. Mao, Y., Yu, L., Yang, R., Ma, C., Qu, L. and Harrington, P.B. (2017) New peptide inhibitors modulate the self-assembly of islet amyloid polypeptide residues 11-20 in vitro. Eur J Pharmacol. 804, 102-110.

56. Fisher, E., Zhao, Y., Richardson, R., Janik, M., Buell, A.K., Aigbirhio, F.I. and Toth, G. (2017) Detection and Characterization of Small Molecule Interactions with Fibrillar Protein Aggregates Using Microscale Thermophoresis. ACS Chem Neurosci. 8, 2088-2095.

57. Wolff, M., Mittag, J.J., Herling, T.W., De Genst, E., Dobson, C.M., Knowles, T.P.J. et al. (2016) Quantitative thermophoretic study of disease-related protein aggregates. Scientific Reports. 6.

58. Mao, Y., Yu, L., Yang, R., Ma, C., Qu, L.B. and Harrington Pde, B. (2016) New insights into side effect of solvents on the aggregation of human islet amyloid polypeptide 11-20. Talanta. 148, 380-386.

59. Jerabek-Willemsen, M., Wienken, C.J., Braun, D., Baaske, P. and Duhr, S. (2011) Molecular interaction studies using microscale thermophoresis. Assay Drug Dev Technol. 9, 342-353.

60. Seidel, S.A., Dijkman, P.M., Lea, W.A., van den Bogaart, G., Jerabek-Willemsen, M., Lazic, A. et al. (2013) Microscale thermophoresis quantifies biomolecular interactions under previously challenging conditions. Methods. 59, 301-315.

61. Zhou, B.R., Zhou, Z., Hu, Q.L., Chen, J. and Liang, Y. (2008) Mixed macromolecular crowding inhibits amyloid formation of hen egg white lysozyme. Biochim Biophys Acta. 1784, 472-480.

62. Daoud, M., Cotton, J.P., Farnoux, B., Jannink, G., Sarma, G., Benoit, H. et al. (1975) Solutions of Flexible Polymers - Neutron Experiments and Interpretation. Macromolecules. 8, 804-818.

63. Cotton, J.P., Nierlich, M., Boue, F., Daoud, M., Farnoux, B., Jannink, G. et al. (1976) Experimental-Determination of Temperature-Concentration Diagram of Flexible Polymer-Solutions by Neutron-Scattering. Journal of Chemical Physics. 65, 11011108.

64. Bolten, D. and Turk, M. (2011) Experimental Study on the Surface Tension, Density, and Viscosity of Aqueous Poly(vinylpyrrolidone) Solutions. J Chem Eng Data. 56, 582-588.

65. Gargallo, L., Leiva, A., Alegria, L., Miranda, B., Gonzalez, A. and Radic, D. (2004) Interfacial properties of poly(N-vinyl-2-pyrrolidone) at the air/water interface. $J$ Macromol Sci Phys. B43, 913-924.

66. Miklos, A.C., Li, C.G., Sharaf, N.G. and Pielak, G.J. (2010) Volume Exclusion and Soft Interaction Effects on Protein Stability under Crowded Conditions. Biochemistry. 49, 6984-6991.

67. Meng, F., Abedini, A., Song, B. and Raleigh, D.P. (2007) Amyloid formation by proislet amyloid polypeptide processing intermediates: examination of the role of protein heparan sulfate interactions and implications for islet amyloid formation in type 2 diabetes. Biochemistry. 46, 12091-12099.

68. Wang, H., Cao, P. and Raleigh, D.P. (2013) Amyloid formation in heterogeneous environments: islet amyloid polypeptide glycosaminoglycan interactions. $J \mathrm{Mol}$ Biol. 425, 492-505.

69. Brender, J.R., Lee, E.L., Hartman, K., Wong, P.T., Ramamoorthy, A., Steel, D.G. and Gafni, A. (2011) Biphasic effects of insulin on islet amyloid polypeptide membrane disruption. Biophys J. 100, 685-692. 
70. Mishra, R., Sorgjerd, K., Nystrom, S., Nordigarden, A., Yu, Y.C. and Hammarstrom, P. (2007) Lysozyme amyloidogenesis is accelerated by specific nicking and fragmentation but decelerated by intact protein binding and conversion. J Mol Biol. 366, 1029-1044.

71. Yamamoto, N., Tsuhara, S., Tamura, A. and Chatani, E. (2018) A specific form of prefibrillar aggregates that functions as a precursor of amyloid nucleation. Sci Rep. 8, 62 . 